\title{
INTEGRAL TRANSFORM APPROACH TO SOLVING KLEIN-GORDON EQUATION WITH VARIABLE COEFFICIENTS
}

\author{
Karen Yagdjian \\ Department of Mathematics \\ University of Texas-Pan American \\ 1201 W. University Drive \\ Edinburg, TX 78539 USA
}

\begin{abstract}
In this paper we describe the integral transform that allows to write solutions of one partial differential equation via solution of another one. This transform was suggested by the author in the case when the last equation is a wave equation, and then it was used to investigate several well-known equations such as Tricomi-type equation, the Klein-Gordon equation in the de Sitter and Einstein-de Sitter spacetimes. A generalization given in this paper allows us to consider also the Klein-Gordon equations with coefficients depending on the spatial variables.
\end{abstract}

Keywords: Klein-Gordon equation; Curved spacetime; Representation of solution

\section{$0 \quad$ Introduction and Statement of Results}

In this paper we give some generalization of the approach suggested in [28], which is aimed to reduce equations with variable coefficients to more simple ones. This transform was used in a series of papers [10, 11], [28][33] to investigate in a unified way several equations such as the linear and semilinear Tricomi equations, Gellerstedt equation, the wave equation in Einstein-de Sitter spacetime, the wave and the Klein-Gordon equations in the de Sitter and anti-de Sitter spacetimes. The listed equations play an important role in the gas dynamics, elementary particle physics, quantum field theory in curved spaces, and cosmology.

Consider for the smooth function $f=f(x, t)$ the solution $w=w_{A, f}(x, t ; b)$ to the problem

$$
v_{t t}-A\left(x, \partial_{x}\right) v=0, v(x, 0 ; b)=f(x, b), v_{t}(x, 0)=0, t \in\left[0, T_{1}\right] \subseteq \mathbb{R}, x \in \Omega \subseteq \mathbb{R}^{n},
$$

with the parameter $b \in I=\left[t_{0}, T\right] \subseteq \mathbb{R}, t_{0}<T \leq \infty$, and with $0<T_{1} \leq \infty$. Here $\Omega$ is a domain in $\mathbb{R}^{n}$, while $A\left(x, \partial_{x}\right)$ is the partial differential operator $A\left(x, \partial_{x}\right)=\sum_{|\alpha| \leq m} a_{\alpha}(x) D_{x}^{\alpha}$. We are going to present the integral operator

$$
\mathcal{K}[w](x, t)=2 \int_{t_{0}}^{t} d b \int_{0}^{|\phi(t)-\phi(b)|} K(t ; r, b ; M) w(x, r ; b) d r, \quad x \in \Omega, t \in I,
$$

which maps the function $w=w(x, r ; b)$ into solution of the equation

$$
u_{t t}-a^{2}(t) A\left(x, \partial_{x}\right) u-M^{2} u=f, \quad x \in \Omega, t \in I .
$$

In fact, the function $u=u(x, t)$ takes initial values as follows

$$
u\left(x, t_{0}\right)=0, u_{t}\left(x, t_{0}\right)=0, \quad x \in \Omega .
$$


Here $\phi=\phi(t)$ is a distance function produced by $a=a(t)$, that is $\phi(t)=\int_{t_{0}}^{t} a(\tau) d \tau$, while $M \in \mathbb{C}$ is a constant. Moreover, we also give the corresponding operators, which generate solutions of the source-free equation and takes non-vanishing initial values. These operators are constructed in $[29,30]$ in the case of $A\left(x, \partial_{x}\right)=\Delta$, where $\Delta$ is the Laplace operator on $\mathbb{R}^{n}$, and, consequently, the equation (0.1) is the wave equation. In the present paper we restrict ourselves to the smooth functions, but it is evident that similar formulas, with the corresponding interpretations, are applicable to the distributions as well. (For details see, e.g., [29].)

In order to motivate our approach, we consider the solution $v=v(x, t ; b)$ to the Cauchy problem

$$
v_{t t}-\Delta v=0,(t, x) \in \mathbb{R}^{1+n}, v(x, 0 ; b)=\varphi(x, b), v_{t}(x, 0)=0, x \in \mathbb{R}^{n},
$$

with the parameter $b \in I \subseteq \mathbb{R}$. We denote that solution by $v_{\varphi}=v_{\varphi}(x, t ; b)$; if $\varphi$ is independent of the second time variable $b$, then we write simply $v_{\varphi}(x, t)$. There are well-known explicit representation formulas for the solution of the problem (0.4). (See, e.g., [24].)

The starting point of the approach suggested in [28] is the Duhamel's principle (see, e.g., [24]), which has been revised in order to prepare the ground for generalization. Our first observation is that the function

$$
u(x, t)=\int_{t_{0}}^{t} d b \int_{0}^{t-b} w_{f}(x, r ; b) d r
$$

is the solution of the Cauchy problem $u_{t t}-\Delta u=f(x, t)$ in $\mathbb{R}^{n+1}$, and $u\left(x, t_{0}\right)=0, u_{t}\left(x, t_{0}\right)=0$ in $\mathbb{R}^{n}$, if the function $w_{f}=w_{f}(x ; t ; b)$ is a solution of the problem $(0.4)$, where $\varphi=f$. The second observation is that in (0.5) the upper limit $t-b$ of the inner integral is generated by the propagation phenomena with the speed which equals to one. In fact, that is a distance function. Our third observation is that the solution operator $\mathcal{G}: f \longmapsto u$ can be regarded as a composition of two operators. The first one

$$
\mathcal{W E}: f \longmapsto w
$$

is a Fourier Integral Operator, which is a solution operator of the Cauchy problem for wave equation. The second operator

$$
\mathcal{K}: w \longmapsto u
$$

is the integral operator given by (0.5). We regard the variable $b$ in (0.5) as a "subsidiary time". Thus, $\mathcal{G}=\mathcal{K} \circ \mathcal{W E}$ and we arrive at the diagram of Figure 1.

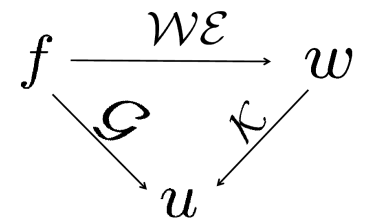

Figure 1: Case of wave equation $A\left(x, \partial_{x}\right)=\Delta$

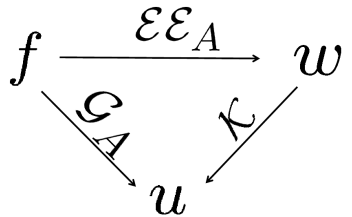

Figure 2: Case of general $A\left(x, \partial_{x}\right)$

Based on the first diagram, we have generated in [31] a class of operators for which we have obtained explicit representation formulas for the solutions, and, in particular, the representations for the fundamental solutions of the partial differential operator. In fact, this diagram brings into a single hierarchy several different partial differential operators. Indeed, if we take into account the propagation cone by introducing the distance function $\phi(t)$, and if we provide the integral operator $(0.5)$ with the kernel $K(t ; r, b ; M)$, as in $(0.2)$, then we actually generate new representations for the solutions of different well-known equations with $x$-independent coefficients. (See, for details, [31].)

In the present paper we extend the class of the equations for which we can obtain explicit representation formulas for the solutions, by varying the first mapping. More precisely, consider the diagram of Figure 2, 
where $w=w_{A, \varphi}(x, t ; b)$ is a solution to the problem $(0.1)$ with the parameter $b \in I \subseteq \mathbb{R}$. If we have a resolving operator of the problem (0.1), then, by applying (0.2), we can generate solutions of another equation. Thus, $\mathcal{G}_{A}=\mathcal{K} \circ \mathcal{E E}_{A}$. The new class of equations contains operators with $x$-depending coefficients, and those equations are not necessarily hyperbolic.

In this paper we restrict ourselves to the Klein-Gordon equation in the de Sitter spacetime, that is $a(t)=e^{-t}$ in (0.1). Recently the equations in the de Sitter and anti-de Sitter spacetimes became the focus of interest for an increasing number of authors (see, e.g., [1]-[3], [6]-[8], [9], [14]-[17], [19] -[21], [23, 26, 27, 34] and the bibliography therein) which investigate those equations from a wide spectrum of perspectives. The creation of a tool for the investigation of the local and global solvability in the problems for these linear and nonlinear equations appears to be a worthwhile undertaking. We believe that the integral transform and the representation formulas for the solutions that we derive in this article fill up the gap in the literature on that topic.

To formulate the main result of this paper we need the following notations. First, we define a chronological future $D_{+}\left(x_{0}, t_{0}\right)$ and a chronological past $D_{-}\left(x_{0}, t_{0}\right)$ of the point $\left(x_{0}, t_{0}\right), x_{0} \in \mathbb{R}^{n}, t_{0} \in \mathbb{R}$, as follows: $D_{ \pm}\left(x_{0}, t_{0}\right):=\left\{(x, t) \in \mathbb{R}^{n+1} ;\left|x-x_{0}\right| \leq \pm\left(e^{-t_{0}}-e^{-t}\right)\right\}$. Then, for $\left(x_{0}, t_{0}\right) \in \mathbb{R}^{n} \times \mathbb{R}, M \in \mathbb{C}$, we define the function

$$
\begin{aligned}
E\left(x, t ; x_{0}, t_{0} ; M\right):= & 4^{-M} e^{M\left(t_{0}+t\right)}\left(\left(e^{-t_{0}}+e^{-t}\right)^{2}-\left(x-x_{0}\right)^{2}\right)^{M-\frac{1}{2}} \\
& \times F\left(\frac{1}{2}-M, \frac{1}{2}-M ; 1 ; \frac{\left(e^{-t_{0}}-e^{-t}\right)^{2}-\left(x-x_{0}\right)^{2}}{\left(e^{-t_{0}}+e^{-t}\right)^{2}-\left(x-x_{0}\right)^{2}}\right),
\end{aligned}
$$

where $(x, t) \in D_{+}\left(x_{0}, t_{0}\right) \cup D_{-}\left(x_{0}, t_{0}\right)$ and $F(a, b ; c ; \zeta)$ is the hypergeometric function. (For definition of the hypergeometric function, see, e.g., [4].) When no ambiguity arises, like in (0.6), we use the notation $x^{2}:=|x|^{2}$ for $x \in \mathbb{R}^{n}$. Thus, the function $E$ depends on $r^{2}=\left(x-x_{0}\right)^{2}$, that is $E\left(x, t ; x_{0}, t_{0} ; M\right)=E\left(r, t ; 0, t_{0} ; M\right)$. According to Theorem 1.12, the function $E\left(r, t ; 0, t_{0} ; M\right)$ solves the following one dimensional Klein-Gordon equation in the de Sitter spacetime:

$$
E_{t t}\left(r, t ; 0, t_{0} ; M\right)-e^{-2 t} E_{r r}\left(r, t ; 0, t_{0} ; M\right)-M^{2} E\left(r, t ; 0, t_{0} ; M\right)=0 .
$$

The kernels $K_{0}(z, t ; M)$ and $K_{0}(z, t ; M)$ are defined by

$$
\begin{aligned}
K_{0}(z, t ; M):= & 4^{-M} e^{t M}\left(\left(1+e^{-t}\right)^{2}-z^{2}\right)^{M} \frac{1}{\left[\left(1-e^{-t}\right)^{2}-z^{2}\right] \sqrt{\left(1+e^{-t}\right)^{2}-z^{2}}} \\
\times & {\left[\left(e^{-t}-1+M\left(e^{-2 t}-1-z^{2}\right)\right) F\left(\frac{1}{2}-M, \frac{1}{2}-M ; 1 ; \frac{\left(1-e^{-t}\right)^{2}-z^{2}}{\left(1+e^{-t}\right)^{2}-z^{2}}\right)\right.} \\
& \quad+\left(1-e^{-2 t}+z^{2}\right)\left(\frac{1}{2}+M\right) F\left(-\frac{1}{2}-M, \frac{1}{2}-M ; 1 ; \frac{\left(1-e^{-t}\right)^{2}-z^{2}}{\left.\left.\left(1+e^{-t}\right)^{2}-z^{2}\right)\right],}\right. \\
K_{1}(z, t ; M):= & 4^{-M} e^{M t}\left(\left(1+e^{-t}\right)^{2}-z^{2}\right)^{-\frac{1}{2}+M} F\left(\frac{1}{2}-M, \frac{1}{2}-M ; 1 ; \frac{\left(1-e^{-t}\right)^{2}-z^{2}}{\left(1+e^{-t}\right)^{2}-z^{2}}\right) .
\end{aligned}
$$

The equation (0.3) is said to be an equation with imaginary (real) mass if there is minus (plus) in front of $M^{2}$; however $M \in \mathbb{C}$. For the Klein-Gordon equation with imaginary mass we have the following result.

Theorem 0.1 For $f \in C^{\infty}(\Omega \times I), I=[0, T], 0<T \leq \infty$, and $\varphi_{0}, \varphi_{1} \in C_{0}^{\infty}(\Omega)$, let the function $v_{f}(x, t ; b)$ be a solution to the problem

$$
\left\{\begin{array}{l}
v_{t t}-A\left(x, \partial_{x}\right) v=0, \quad x \in \Omega, \quad t \in\left[0,1-e^{-T}\right] \\
v(x, 0 ; b)=f(x, b), \quad v_{t}(x, 0 ; b)=0, \quad b \in I, \quad x \in \Omega,
\end{array}\right.
$$

and the function $v_{\varphi}=v_{\varphi}(x, t)$ be a solution of the problem

$$
\left\{\begin{array}{l}
v_{t t}-A\left(x, \partial_{x}\right) v=0, \quad x \in \Omega, \quad t \in\left[0,1-e^{-T}\right] \\
v(x, 0)=\varphi(x), \quad v_{t}(x, 0)=0, \quad x \in \Omega
\end{array}\right.
$$


Then the function $u=u(x, t)$ defined by

$$
\begin{aligned}
u(x, t)= & 2 \int_{0}^{t} d b \int_{0}^{\phi(t)-\phi(b)} v_{f}(x, r ; b) E(r, t ; 0, b ; M) d r+e^{\frac{t}{2}} v_{\varphi_{0}}(x, \phi(t)) \\
& +2 \int_{0}^{\phi(t)} v_{\varphi_{0}}(x, s) K_{0}(s, t ; M) d s+2 \int_{0}^{\phi(t)} v_{\varphi_{1}}(x, s) K_{1}(s, t ; M) d s, \quad x \in \Omega, t \in I,
\end{aligned}
$$

where $\phi(t):=1-e^{-t}$, solves the problem

$$
\left\{\begin{array}{l}
u_{t t}-e^{-2 t} A\left(x, \partial_{x}\right) u-M^{2} u=f, \quad x \in \Omega, t \in I, \\
u(x, 0)=\varphi_{0}(x), \quad u_{t}(x, 0)=\varphi_{1}(x), \quad x \in \Omega .
\end{array}\right.
$$

Here the kernels $E, K_{0}$ and $K_{1}$ have been defined in (0.6), (0.7) and (0.8), respectively.

We note that the operator $A\left(x, \partial_{x}\right)$ is of arbitrary order, that is, the equation of $(0.12)$ can be an evolution equation, not necessarily hyperbolic. Then, the problems in $(0.9)$ and $(0.12)$ can be a mixed initial-boundary value problem involving the boundary condition. Indeed, assume that $\Omega \subset \mathbb{R}^{n}$ is domain with smooth boundary $\partial \Omega$, and that $\nu=\nu(x)$ is a unit normal vector. Let $\alpha=\alpha(x)$ and $\beta=\beta(x)$ be continuous functions, $\alpha, \beta \in C(\partial \Omega)$. If $v=v(t, x)$ satisfies the boundary condition

$$
\alpha(x) v(x, t)+\beta(x) \partial_{\nu} v(x, t)=0 \quad \text { for all } t \in\left[0,1-e^{T}\right], \quad x \in \partial \Omega,
$$

then the function $u=u(x, t)$ fulfills the same boundary condition

$$
\alpha(x) u(x, t)+\beta(x) \partial_{\nu} u(x, t)=0 \quad \text { for all } \quad t \in I, \quad x \in \partial \Omega .
$$

Next, we stress that interval $\left[0,1-e^{-T}\right] \subseteq[0,1]$, which appears in (0.9), reflects the fact that de Sitter model possesses the horizon [12]; existence of the horizon in the de Sitter model is widely used to define an asymptotically de Sitter space (see, e.g., $[2,26]$ ) and to involve geometry into the analysis of the operators on the de Sitter space (see, e.g., $[5,19,22,25])$.

Although the next statement is a straightforward consequence of Theorem 0.1 , we present it as a separate theorem having in mind the importance of the equation with real mass. In the next theorem we use the following kernels

$$
\begin{aligned}
E\left(x, t ; x_{0}, t_{0}\right) & :=E\left(x, t ; x_{0}, t_{0} ;-i M\right), \\
K_{0}(z, t) & :=K_{0}(z, t ;-i M), \\
K_{1}(z, t) & :=K_{1}(z, t ;-i M),
\end{aligned}
$$

which were introduced in [29].

Theorem 0.2 For $f \in C^{\infty}(\Omega \times I), I=[0, T], 0<T \leq \infty$, and $\varphi_{0}, \varphi_{1} \in C_{0}^{\infty}(\Omega)$, let the function $v_{f}(x, t ; b)$ be a solution to the problem

$$
\left\{\begin{array}{l}
v_{t t}-A\left(x, \partial_{x}\right) v=0, \quad x \in \Omega, \quad t \in\left[0,1-e^{-T}\right], \\
v(x, 0 ; b)=f(x, b), \quad v_{t}(x, 0 ; b)=0, \quad b \in I, \quad x \in \Omega,
\end{array}\right.
$$

and the function $v_{\varphi}=v_{\varphi}(x, t)$ be a solution of the problem

$$
\left\{\begin{array}{l}
v_{t t}-A\left(x, \partial_{x}\right) v=0, \quad x \in \Omega, \quad t \in\left[0,1-e^{-T}\right] \\
v(x, 0)=\varphi(x), \quad v_{t}(x, 0)=0, \quad x \in \Omega
\end{array}\right.
$$

Then the function $u=u(x, t)$ defined by

$$
\begin{aligned}
u(x, t)= & 2 \int_{0}^{t} d b \int_{0}^{\phi(t)-\phi(b)} v_{f}(x, r ; b) E(r, t ; 0, b) d r+e^{\frac{t}{2}} v_{\varphi_{0}}(x, \phi(t)) \\
& +2 \int_{0}^{\phi(t)} v_{\varphi_{0}}(x, s) K_{0}(s, t) d s+2 \int_{0}^{\phi(t)} v_{\varphi_{1}}(x, s) K_{1}(s, t) d s, \quad x \in \Omega, t \in I,
\end{aligned}
$$


where $\phi(t):=1-e^{-t}$, solves the problem

$$
\left\{\begin{array}{l}
u_{t t}-e^{-2 t} A\left(x, \partial_{x}\right) u+M^{2} u=f, \quad x \in \Omega, t \in I \\
u(x, 0)=\varphi_{0}(x), \quad u_{t}(x, 0)=\varphi_{1}(x), \quad x \in \Omega
\end{array}\right.
$$

Here the kernels $E, K_{0}$ and $K_{1}$ have been defined in (0.13), (0.14) and (0.15), respectively.

The special cases of Theorems $0.1,0.2$, when $A\left(x, \partial_{x}\right)=\Delta$, one can find in [29,30]. The proof given in those papers is based on the well-known explicit representation formulas for the wave equation, the Riemann function, the spherical means, and the Asgeirsson's mean value theorem. The main outcome, resulting from the application of all those tools, is the derivation of the final representation formula and the kernels $E, K_{0}$, and $K_{1}$. Having in the hand the integral transform and the final formulas, we suggest here straightforward proof by substitution, which works also for the equations with coefficients depending on $x$.

Among possible applications of the integral transform method are the $L^{p}-L^{q}$ estimates, Strichartz estimates, Huygens' principle, global and local existence theorem for semilinear and quasilinear equations. Below we give examples of the equations with the variable coefficients those are amenable to the integral transform method.

Example 1. The metric $g$ in the de Sitter type spacetime, that is, $g_{00}=g^{00}=-1, g_{0 j}=g^{0 j}=0, g_{i j}(x, t)=$ $e^{2 t} \delta_{i j}(x),|g(x, t)|=e^{2 n t}|\operatorname{det} \delta(x)|, g^{i j}(x, t)=e^{-2 t} \delta^{i j}(x), i, j=1,2, \ldots, n$, where $\sum_{j=1}^{n} \delta^{i j}(x) \delta_{j k}(x)=\delta_{i k}$, and $\delta_{i j}$ is Kronecker's delta. The linear covariant Klein-Gordon equation in the coordinates is

$$
\psi_{t t}-\frac{e^{-2 t}}{\sqrt{|\operatorname{det} \delta(x)|}} \sum_{i, j=1}^{n} \frac{\partial}{\partial x^{i}}\left(\sqrt{|\operatorname{det} \delta(x)|} \delta^{i j}(x) \frac{\partial}{\partial x^{j}} \psi\right)+n \psi_{t}+m^{2} \psi=f .
$$

Here $m$ is a physical mass of the particle. If we introduce the new unknown function $u=e^{n t / 2} \psi$, then the equation takes the form of the Klein-Gordon equation with imaginary mass

$$
u_{t t}-\frac{e^{-2 t}}{\sqrt{|\operatorname{det} \delta(x)|}} \sum_{i, j=1}^{n} \frac{\partial}{\partial x^{i}}\left(\sqrt{|\operatorname{det} \delta(x)|} \delta^{i j}(x) \frac{\partial}{\partial x^{j}} u\right)-M^{2} u=f,
$$

where $-M^{2}=m^{2}-\frac{n^{2}}{4}$ is the square of the so-called curved (or effective) mass. For the last equation we set

$$
A\left(x, \partial_{x}\right) u=\frac{1}{\sqrt{|\operatorname{det} \delta(x)|}} \sum_{i, j=1}^{n} \frac{\partial}{\partial x^{i}}\left(\sqrt{|\operatorname{det} \delta(x)|} \delta^{i j}(x) \frac{\partial}{\partial x^{j}} u\right) .
$$

If $\Omega=\Pi$ is a non-Euclidean space of constant negative curvature and the equation of the problems $(0.9)$ and $(0.10)$ is a non-Euclidean wave equation, then the explicit representation formulas are known (see, e.g., $[13,18])$ and the Huygens' principle is a consequence of those formulas. Thus, for a non-Euclidean wave equation, due to Theorem 0.1 , the functions $v_{f}(x, t ; b)$ and $v_{\varphi}(x, t)$ have explicit representations, and the arguments of $[29,33]$ allow us to derive for the solution $u(x, t)$ of the problem $(0.12)$ in the de Sitter type metric with hyperbolic spatial geometry the explicit representation, the $L^{p}-L^{q}$ estimates, and to examine the Huygens' principle. Precise statements will be published in the forthcoming paper.

Example 2. The Euler-Bernoulli beam equation with the variable coefficients

$$
\psi_{t t}+e^{-2 t} \sum_{i, j=1}^{n} \partial_{x_{i}}^{2}\left(a^{i j}(x) \partial_{x_{j}}^{2} \psi\right)=f
$$

Here $A\left(x, \partial_{x}\right)=\sum_{i, j=1}^{n} \partial_{x_{i}}^{2} a^{i j}(x) \partial_{x_{j}}^{2}$ and we assume that $\sum_{i, j=1}^{n} a^{i j}(x) \xi_{i} \xi_{j} \geq 0$.

This paper is organized as follows. In Section 1 we study the kernel functions $E, K_{0}$, and $K_{1}$, and prove several basic properties of those function. Then, in Section 2, we prove Theorem 0.1. Applications of Theorems $0.1,0.2$ to some equations appearing in electrodynamics and cosmology will be done in a forthcoming paper. 


\section{The Kernels of the Integral Transforms}

The proof of Theorem 0.1 is straightforward; we just substitute the function of (0.11) in the equation of (0.12), and then check the initial conditions. It is straightforward, but not short; it requires very long and tedious calculations. In order to make the calculations more transparent, we reveal in this section several main properties of the kernel functions.

\subsection{The kernel function $E(r, t ; 0, b ; M)$}

In this subsection we collect some important properties of the kernel $E\left(x, t ; x_{0}, t_{0} ; M\right)$. Although the function $E\left(x, t ; x_{0}, t_{0} ; M\right)(0.6)$ is defined for $x, x_{0} \in \mathbb{R}^{n}$, we use it for $x_{0}=0$ and $x=r \in \mathbb{R}$, only. Consider for $t, b \in \mathbb{R}, 0 \leq b \leq t, M \in \mathbb{C}, r \in\left[0, e^{-b}-e^{-t}\right]$ the function

$$
E(r, t ; 0, b ; M):=4^{-M} e^{M(b+t)}\left(\left(e^{-b}+e^{-t}\right)^{2}-r^{2}\right)^{M-\frac{1}{2}} F\left(\frac{1}{2}-M, \frac{1}{2}-M ; 1 ; \frac{\left(e^{-b}-e^{-t}\right)^{2}-r^{2}}{\left(e^{-b}+e^{-t}\right)^{2}-r^{2}}\right) .
$$

The following notations are helpful to evaluate derivatives of the kernel functions

$$
\begin{aligned}
\alpha(r, t, b ; M) & :=4^{-M} e^{M(b+t)}\left(\left(e^{-b}+e^{-t}\right)^{2}-r^{2}\right)^{M}, \\
\beta(r, t, b) & :=\left(\left(e^{-b}+e^{-t}\right)^{2}-r^{2}\right)^{-\frac{1}{2}} \\
\gamma(r, t, b) & :=\frac{\left(e^{-b}-e^{-t}\right)^{2}-r^{2}}{\left(e^{-b}+e^{-t}\right)^{2}-r^{2}} .
\end{aligned}
$$

We rewrite the function $E(r, t ; 0, b ; M)$ in terms of these functions as follows

$$
E(r, t ; 0, b ; M)=\alpha(r, t, b ; M) \beta(r, t, b) F\left(\frac{1}{2}-M, \frac{1}{2}-M ; 1 ; \gamma(r, t, b)\right) .
$$

For the derivatives of the auxiliary functions $\alpha, \beta$, and $\gamma$ we have

Lemma 1.1 The partial derivatives of the functions $\alpha, \beta$, and $\gamma$ are as follows

$$
\begin{aligned}
\alpha_{r}(r, t, b ; M) & =-2 M r \alpha(r, t, b ; M) \beta^{2}(r, t, b) \\
\beta_{r}(r, t, b) & =r \beta^{3}(r, t, b) \\
\gamma_{r}(r, t, b) & =2 r \beta^{2}(r, t, b)[\gamma(r, t, b)-1] \\
\alpha_{r r}(r, t, b ; M) & =-2 M \alpha(r, t, b ; M) \beta^{2}(r, t, b)\left[1-2 M r^{2} \beta^{2}(r, t, b)+4 r \beta^{2}(r, t, b)\right] \\
\beta_{r r}(r, t, b) & =\beta^{3}(r, t, b)+3 r^{2} \beta^{5}(r, t, b) \\
\gamma_{r r}(r, t, b) & =2 \beta^{2}(r, t, b)\left(1+4 r^{2} \beta^{2}(r, t, b)\right)[\gamma(r, t, b)-1] .
\end{aligned}
$$

Proof. We skip the simple proof of formulas for the first-order derivatives and of $\beta_{r r}(r, t, b)$. For the second-order derivatives we have

$$
\begin{aligned}
\alpha_{r r}(r, t, b ; M)= & -2 M \alpha(r, t, b ; M) \beta^{2}(r, t, b)+4 M^{2} r^{2} \alpha(r, t, b ; M) \beta^{4}(r, t, b) \\
& -4 M r^{2} \alpha(r, t, b ; M) \beta^{4}(r, t, b) \\
= & -2 M \alpha(r, t, b ; M) \beta^{2}(r, t, b)\left[1-2 M r^{2} \beta^{2}(r, t, b)+4 r \beta^{2}(r, t, b)\right], \\
\gamma_{r r}(r, t, b)= & 2 \beta^{2}(r, t, b)[\gamma(r, t, b)-1]+4 r \beta_{r}(r, t, b) \beta(r, t, b)[\gamma(r, t, b)-1] \\
& +2 r \beta^{2}(r, t, b) \gamma_{r}(r, t, b) \\
= & 2 \beta^{2}(r, t, b)[\gamma(r, t, b)-1]+4 r^{2} \beta^{4}(r, t, b)[\gamma(r, t, b)-1] \\
& +4 r^{2} \beta^{4}(r, t, b)[\gamma(r, t, b)-1] \\
= & 2 \beta^{2}(r, t, b)\left(1+4 r^{2} \beta^{2}(r, t, b)\right)[\gamma(r, t, b)-1] .
\end{aligned}
$$

Lemma is proven. 
Corollary 1.2 The derivatives, explicitly written, are as follows:

$$
\begin{aligned}
\alpha_{r}(r, t, b ; M) & =-2^{1-2 M} M r e^{M(b+t)}\left(\left(e^{-b}+e^{-t}\right)^{2}-r^{2}\right)^{M-1} \\
\beta_{r}(r, t, b) & =r\left(\left(e^{-b}+e^{-t}\right)^{2}-r^{2}\right)^{-3 / 2} \\
\gamma_{r}(r, t, b) & =-8 r e^{3(b+t)}\left(\left(e^{b}+e^{t}\right)^{2}-r^{2} e^{2(b+t)}\right)^{-2}
\end{aligned}
$$

In particular, for $r=e^{-b}-e^{-t}$ we obtain the next values of those functions.

Lemma 1.3 For the functions $\alpha, \beta$, and $\gamma$ we have

$$
\alpha\left(e^{-b}-e^{-t}, t, b ; M\right)=1, \quad \beta\left(e^{-b}-e^{-t}, t, b\right)=2^{-1} e^{\frac{1}{2}(b+t)}, \quad \gamma\left(e^{-b}-e^{-t}, t, b\right)=0,
$$

while for their derivatives we have

$$
\begin{aligned}
\alpha_{r}\left(e^{-b}-e^{-t}, t, b ; M\right) & =-2^{-1} M\left(e^{-b}-e^{-t}\right) e^{b+t}, \\
\beta_{r}\left(e^{-b}-e^{-t}, t, b\right) & =2^{-3}\left(e^{-b}-e^{-t}\right) e^{\frac{3}{2}(b+t)}, \\
\gamma_{r}\left(e^{-b}-e^{-t}, t, b\right) & =-2^{-1}\left(e^{-b}-e^{-t}\right) e^{b+t} .
\end{aligned}
$$

Proof. It is a simple consequence of the definitions of these functions and the previous lemma. Lemma is proven.

Now we turn to the derivatives of the function $E(r, t ; 0, b ; M)$.

Proposition 1.4 The derivative $E_{r}(r, t ; 0, b ; M):=\partial_{r} E(r, t ; 0, b ; M)$ is given as follows

$$
\begin{array}{r}
\partial_{r} E(r, t ; 0, b ; M)=2\left(\frac{1}{2}-M\right) r \alpha(r, t, b ; M) \beta^{3}(r, t, b)\left[F\left(\frac{1}{2}-M, \frac{1}{2}-M ; 1 ; \gamma(r, t, b)\right)\right. \\
\left.+(\gamma(r, t, b)-1)\left(\frac{1}{2}-M\right) F\left(\frac{3}{2}-M, \frac{3}{2}-M ; 2 ; \gamma(r, t, b)\right)\right] .
\end{array}
$$

Moreover,

$$
\begin{aligned}
E_{r}(0, t ; 0, b ; M) & =0 \\
E_{r}\left(e^{-b}-e^{-t}, t ; 0, b ; M\right) & =2^{-2}\left(\frac{1}{4}-M^{2}\right)\left(e^{t}-e^{b}\right) e^{\frac{1}{2}(b+t)} .
\end{aligned}
$$

The second-order derivative $E_{r r}(r, t ; 0, b ; M):=\partial_{r}^{2} E(r, t ; 0, b ; M)$ is given by

$$
\begin{aligned}
\partial_{r}^{2} E(r, t ; 0, b ; M)= & 2\left(\frac{1}{2}-M\right) \alpha(r, t, b ; M) \beta^{3}(r, t, b)\left[1+2\left(\frac{3}{2}-M\right) r^{2} \beta^{2}(r, t, b)\right] \\
\times & {\left[F\left(\frac{1}{2}-M, \frac{1}{2}-M ; 1 ; \gamma(r, t, b)\right)\right.} \\
& \left.+(\gamma(r, t, b)-1)\left(\frac{1}{2}-M\right) F\left(\frac{3}{2}-M, \frac{3}{2}-M ; 2 ; \gamma(r, t, b)\right)\right] \\
+ & 4\left(\frac{1}{2}-M\right)^{2}\left(\frac{3}{2}-M\right) r^{2} \alpha(r, t, b ; M) \beta^{5}(r, t, b)(\gamma(r, t, b)-1) \\
\times & {\left[F\left(\frac{3}{2}-M, \frac{3}{2}-M ; 2 ; \gamma(r, t, b)\right)\right.} \\
& \left.+(\gamma(r, t, b)-1)\left(\frac{3}{2}-M\right) \frac{1}{2} F\left(\frac{5}{2}-M, \frac{5}{2}-M ; 3 ; \gamma(r, t, b)\right)\right] .
\end{aligned}
$$


Proof. Indeed, due to [4, (7) Sec. 2.1.2], we have

$$
\begin{aligned}
& \partial_{r} E(r, t ; 0, b ; M) \\
=\quad & {\left[\alpha_{r}(r, t, b ; M) \beta(r, t, b)+\alpha(r, t, b ; M) \beta_{r}(r, t, b)\right] F\left(\frac{1}{2}-M, \frac{1}{2}-M ; 1 ; \gamma(r, t, b)\right) } \\
& +\alpha(r, t, b ; M) \beta(r, t, b) \gamma_{r}(r, t, b)\left(\frac{1}{2}-M\right)^{2} F\left(\frac{3}{2}-M, \frac{3}{2}-M ; 2 ; \gamma(r, t, b)\right) \\
= & {\left[-2 M r \alpha(r, t, b ; M) \beta^{3}(r, t, b)\right.} \\
& \left.+\alpha(r, t, b ; M) r \beta^{3}(r, t, b)\right] F\left(\frac{1}{2}-M, \frac{1}{2}-M ; 1 ; \gamma(r, t, b)\right) \\
& +2 r \alpha(r, t, b ; M) \beta^{3}(r, t, b)[\gamma(r, t, b)-1]\left(\frac{1}{2}-M\right)^{2} F\left(\frac{3}{2}-M, \frac{3}{2}-M ; 2 ; \gamma(r, t, b)\right) .
\end{aligned}
$$

This proves the first formula of the lemma and the statement for $E_{r}\left(e^{-b}-e^{-t}, t ; 0, b ; M\right)$. Then, for the second-order derivative $\partial_{r}^{2} E(r, t ; 0, b ; M)$ we have

$$
\begin{aligned}
& \partial_{r}^{2} E(r, t ; 0, b ; M) \\
= & 2\left(\frac{1}{2}-M\right)\left\{\alpha(r, t, b ; M) \beta^{3}(r, t, b)+r \alpha_{r}(r, t, b ; M) \beta^{3}(r, t, b)\right. \\
& \left.+3 r \alpha(r, t, b ; M) \beta_{r}(r, t, b) \beta^{2}(r, t, b)\right\} \\
& \times\left[F\left(\frac{1}{2}-M, \frac{1}{2}-M ; 1 ; \gamma(r, t, b)\right)\right. \\
& \left.+(\gamma(r, t, b)-1)\left(\frac{1}{2}-M\right) F\left(\frac{3}{2}-M, \frac{3}{2}-M ; 2 ; \gamma(r, t, b)\right)\right] \\
+ & 4\left(\frac{1}{2}-M\right) r^{2} \alpha(r, t, b ; M) \beta^{5}(r, t, b)[\gamma(r, t, b)-1] \\
& \times\left[F_{z}\left(\frac{1}{2}-M, \frac{1}{2}-M ; 1 ; \gamma(r, t, b)\right)+\left(\frac{1}{2}-M\right) F\left(\frac{3}{2}-M, \frac{3}{2}-M ; 2 ; \gamma(r, t, b)\right)\right. \\
+ & \left.(\gamma(r, t, b)-1)\left(\frac{1}{2}-M\right) F_{z}\left(\frac{3}{2}-M, \frac{3}{2}-M ; 2 ; \gamma(r, t, b)\right)\right] \\
= & \left(\frac{1}{2}-M\right)\left\{\alpha(r, t, b ; M) \beta^{3}(r, t, b)-2 M r^{2} \alpha(r, t, b ; M) \beta^{5}(r, t, b)+3 r^{2} \alpha(r, t, b ; M) \beta^{5}(r, t, b)\right\} \\
+ & {\left[F\left(\frac{1}{2}-M, \frac{1}{2}-M ; 1 ; \gamma(r, t, b)\right)\right.} \\
+ & \left.+(\gamma(r, t, b)-1)\left(\frac{1}{2}-M\right) F\left(\frac{3}{2}-M, \frac{3}{2}-M ; 2 ; \gamma(r, t, b)\right)\right] \\
& \times\left[F\left(\frac{3}{2}-M, \frac{3}{2}-M ; 2 ; \gamma(r, t, b)\right)\right. \\
& {\left[\frac{3}{2}-M\right) r^{2} \alpha(r, t, b ; M) \beta^{5}(r, t, b)(\gamma(r, t, b)-1) }
\end{aligned}
$$


It is easily seen that the last expression coincides with one given in the statement of the proposition. This completes the proof of the proposition.

In fact, Corollary 1.2 allows us to write $\partial_{r} E(r, t ; 0, b ; M)$ and $\partial_{r}^{2} E(r, t ; 0, b ; M)$ in the explicit form.

Corollary 1.5 We have

$$
\begin{aligned}
\partial_{r} E(r, t ; 0, b ; M)= & 2\left(\frac{1}{2}-M\right) r 4^{-M} e^{M(b+t)}\left(\left(e^{-t}+e^{-b}\right)^{2}-r^{2}\right)^{M-\frac{3}{2}} \\
\times & {\left[F\left(\frac{1}{2}-M, \frac{1}{2}-M ; 1 ; \frac{\left(e^{-b}-e^{-t}\right)^{2}-r^{2}}{\left(e^{-b}+e^{-t}\right)^{2}-r^{2}}\right)\right.} \\
& \left.\quad-\frac{4 e^{-b-t}}{\left(e^{-b}+e^{-t}\right)^{2}-r^{2}}\left(\frac{1}{2}-M\right) F\left(\frac{3}{2}-M, \frac{3}{2}-M ; 2 ; \frac{\left(e^{-b}-e^{-t}\right)^{2}-r^{2}}{\left(e^{-b}+e^{-t}\right)^{2}-r^{2}}\right)\right], \\
\partial_{r}^{2} E(r, t ; 0, b ; M)= & \widetilde{A}(r, t, b ; M) F\left(\frac{1}{2}-M, \frac{1}{2}-M ; 1 ; \gamma(r, t, b)\right) \\
+ & \widetilde{B}(r, t, b ; M) F\left(\frac{3}{2}-M, \frac{3}{2}-M ; 2 ; \gamma(r, t, b)\right) \\
+ & \widetilde{C}(r, t, b ; M) F\left(\frac{5}{2}-M, \frac{5}{2}-M ; 3 ; \gamma(r, t, b)\right),
\end{aligned}
$$

where

$$
\begin{aligned}
& \widetilde{A}(r, t, b ; M)=2^{1-2 M}\left(\frac{1}{2}-M\right) e^{M(b+t)}\left(\left(e^{-b}+e^{-t}\right)^{2}-r^{2}\right)^{M-\frac{5}{2}}\left[\left(e^{-b}+e^{-t}\right)^{2}+(2-2 M) r^{2}\right], \\
& \widetilde{B}(r, t, b ; M)=-2^{3-2 M}\left(\frac{1}{2}-M\right)^{2} e^{(M-1)(b+t)}\left(\left(e^{-b}+e^{-t}\right)^{2}-r^{2}\right)^{M-\frac{7}{2}}\left[\left(e^{-b}+e^{-t}\right)^{2}+(5-4 M) r^{2}\right], \\
& \widetilde{C}(r, t, b ; M)=2^{5-2 M}\left(\frac{1}{2}-M\right)^{2}\left(\frac{3}{2}-M\right)^{2} e^{(M-2)(b+t)} r^{2}\left(\left(e^{-b}+e^{-t}\right)^{2}-r^{2}\right)^{M-\frac{9}{2}} .
\end{aligned}
$$

Next, we turn to the derivatives with respect to time. We skip the proof of the following lemma, which gives the derivatives of the auxiliary functions $\alpha, \beta$, and $\gamma$.

Lemma 1.6 For the first-order derivatives of the functions $\alpha, \beta$, and $\gamma$ we have

$$
\begin{aligned}
\alpha_{t}(r, t, b ; M) & =M \alpha(r, t, b ; M)-2 M e^{-t}\left(e^{-b}+e^{-t}\right) \alpha(r, t, b ; M) \beta^{2}(r, t, b), \\
\beta_{t}(r, t, b) & =e^{-t}\left(e^{-b}+e^{-t}\right) \beta^{3}(r, t, b), \\
\gamma_{t}(r, t, b) & =2 e^{-t} \beta^{2}(r, t, b)\left\{\left(e^{-b}-e^{-t}\right)+\left(e^{-b}+e^{-t}\right) \gamma(r, t, b)\right\},
\end{aligned}
$$

and

$$
\begin{aligned}
\alpha_{t}\left(e^{-b}-e^{-t}, t, b ; M\right) & =M-2^{-1} M\left(e^{-b}+e^{-t}\right) e^{b}, \\
\beta_{t}\left(e^{-b}-e^{-t}, t, b\right) & =2^{-3} e^{-t}\left(e^{-b}+e^{-t}\right) e^{\frac{3}{2}(b+t)}, \\
\gamma_{t}\left(e^{-b}-e^{-t}, t, b\right) & =2^{-1} e^{-t} e^{(b+t)}\left(e^{-b}-e^{-t}\right) .
\end{aligned}
$$

Proposition 1.7 One can write

$$
\begin{aligned}
\partial_{t} E(r, t ; 0, b ; M)= & A(r, t, b ; M) F\left(\frac{1}{2}-M, \frac{1}{2}-M ; 1 ; \gamma(r, t, b)\right) \\
& +B(r, t, b ; M) F\left(\frac{3}{2}-M, \frac{3}{2}-M ; 2 ; \gamma(r, t, b)\right),
\end{aligned}
$$


where

$$
\begin{aligned}
& A(r, t, b ; M)=\alpha(r, t, b ; M) \beta(r, t, b)\left\{M+2\left(\frac{1}{2}-M\right) e^{-t}\left(e^{-b}+e^{-t}\right) \beta^{2}(r, t, b)\right\} . \\
& B(r, t, b ; M)=2 e^{-t} \alpha(r, t, b ; M) \beta^{3}(r, t, b)\left\{\left(e^{-b}-e^{-t}\right)+\left(e^{-b}+e^{-t}\right) \gamma(r, t, b)\right\} .
\end{aligned}
$$

Proof. Indeed, it is easily seen that

$$
\begin{aligned}
\partial_{t} E(r, t ; 0, b ; M)= & \alpha(r, t, b ; M) \beta(r, t, b)\left(\left\{1-2 e^{-t}\left(e^{-b}+e^{-t}\right) \beta^{2}(r, t, b)\right\} M\right. \\
& \left.+e^{-t}\left(e^{-b}+e^{-t}\right) \beta^{2}(r, t, b)\right) F\left(\frac{1}{2}-M, \frac{1}{2}-M ; 1 ; \gamma(r, t, b)\right) \\
& +2 e^{-t} \alpha(r, t, b ; M) \beta^{3}(r, t, b)\left\{\left(e^{-b}-e^{-t}\right)+\left(e^{-b}+e^{-t}\right) \gamma(r, t, b)\right\} \\
& \times\left(\frac{1}{2}-M\right)^{2} F\left(\frac{3}{2}-M, \frac{3}{2}-M ; 2 ; \gamma(r, t, b)\right) \\
= & \alpha(r, t, b ; M) \beta(r, t, b)\left(M+2\left(\frac{1}{2}-M\right) e^{-t}\left(e^{-b}+e^{-t}\right) \beta^{2}(r, t, b)\right) \\
& \times F\left(\frac{1}{2}-M, \frac{1}{2}-M ; 1 ; \gamma(r, t, b)\right) \\
& +2 e^{-t} \alpha(r, t, b ; M) \beta^{3}(r, t, b)\left\{\left(e^{-b}-e^{-t}\right)+\left(e^{-b}+e^{-t}\right) \gamma(r, t, b)\right\} \\
& \times\left(\frac{1}{2}-M\right)^{2} F\left(\frac{3}{2}-M, \frac{3}{2}-M ; 2 ; \gamma(r, t, b)\right)
\end{aligned}
$$

and, consequently,

$$
\begin{aligned}
& \partial_{t} E(r, t ; 0, b ; M) \\
= & -2^{1-2 M}\left(M-\frac{1}{2}\right)\left(e^{-b}+e^{-t}\right) e^{M(b+t)-t}\left(\left(e^{-b}+e^{-t}\right)^{2}-r^{2}\right)^{M-\frac{3}{2}} \\
& \times F\left(\frac{1}{2}-M, \frac{1}{2}-M ; 1 ; \gamma(r, t, b)\right)+ \\
& +4^{-M} M e^{M(b+t)}\left(\left(e^{-b}+e^{-t}\right)^{2}-r^{2}\right)^{M-\frac{1}{2}} F\left(\frac{1}{2}-M, \frac{1}{2}-M ; 1 ; \gamma(r, t, b)\right) \\
& +4^{-M}\left(\frac{1}{2}-M\right)^{2} e^{M(b+t)}\left(\frac{2 e^{-t}\left(e^{-b}-e^{-t}\right)}{\left(e^{-b}+e^{-t}\right)^{2}-r^{2}}+\frac{2 e^{-t}\left(e^{-b}+e^{-t}\right)\left(\left(e^{-b}-e^{-t}\right)^{2}-r^{2}\right)}{\left(\left(e^{-b}+e^{-t}\right)^{2}-r^{2}\right)^{2}}\right) \\
& \times\left(\left(e^{-b}+e^{-t}\right)^{2}-r^{2}\right)^{M-\frac{1}{2}} F\left(\frac{3}{2}-M, \frac{3}{2}-M ; 2 ; \gamma(r, t, b)\right) .
\end{aligned}
$$

Proposition is proven.

Corollary 1.8 The coefficients $A(r, t, b ; M)$ and $B(r, t, b ; M)$, explicitly written, are as follows

$$
\begin{aligned}
A(r, t, b ; M)= & 2^{-2 M} e^{M(b+t)}\left(\left(e^{-b}+e^{-t}\right)^{2}-r^{2}\right)^{M-\frac{3}{2}} \\
& \times\left[M\left(\left(e^{-b}+e^{-t}\right)^{2}-r^{2}\right)-(2 M-1)\left(e^{-b}+e^{-t}\right) e^{-t}\right],
\end{aligned}
$$




$$
\begin{aligned}
B(r, t, b ; M)= & 2^{1-2 M}\left(\frac{1}{2}-M\right)^{2} e^{M(b+t)} e^{-t}\left(\left(e^{-b}+e^{-t}\right)^{2}-r^{2}\right)^{M-\frac{5}{2}} \\
& \times\left[\left(e^{-b}-e^{-t}\right)\left(e^{-b}+e^{-t}\right)^{2}-\left(e^{-b}-e^{-t}\right) r^{2}\right. \\
& \left.+\left(e^{-b}+e^{-t}\right)\left(e^{-b}-e^{-t}\right)^{2}-\left(e^{-b}+e^{-t}\right) r^{2}\right] .
\end{aligned}
$$

In particular,

$$
E_{t}\left(e^{-b}-e^{-t}, t ; 0, b ; M\right)=\frac{1}{16} e^{\frac{1}{2}(b-t)}\left(e^{b}\left(1-4 M^{2}\right)+\left(4 M^{2}+3\right) e^{t}\right) .
$$

Then, the following proposition will be used.

Proposition 1.9 For all $t, b, M, b \leq t$, we have

$$
2 E_{r}\left(e^{-b}-e^{-t}, t ; 0, b ; M\right)+2 e^{t} E_{t}\left(e^{-b}-e^{-t}, t ; 0, b ; M\right)-e^{t}\left(4 e^{-b-t}\right)^{-\frac{1}{2}}=0 .
$$

Proof. According to Proposition 1.4 and (1.2), we can write

$$
\begin{aligned}
& 2 E_{r}\left(e^{-b}-e^{-t}, t ; 0, b ; M\right)+2 e^{t} E_{t}\left(e^{-b}-e^{-t}, t ; 0, b ; M\right)-e^{t}\left(4 e^{-b-t}\right)^{-\frac{1}{2}} \\
= & \frac{1}{8}\left(4 M^{2}-1\right)\left(e^{b}-e^{t}\right) e^{M(b+t)}\left(e^{-b-t}\right)^{M-\frac{1}{2}} \\
& +\frac{1}{8} e^{M(b+t)}\left(e^{-b-t}\right)^{M-\frac{1}{2}}\left(e^{b}\left(1-4 M^{2}\right)+\left(4 M^{2}-1\right) e^{t}+4 e^{t}\right)-e^{t}\left(4 e^{-b-t}\right)^{-\frac{1}{2}}=0 .
\end{aligned}
$$

Proposition is proven.

Proposition 1.10 For all $r, t, b, M, b \leq t$, we have

$$
\begin{aligned}
\partial_{t}^{2} E(r, t ; 0, b ; M)= & A_{t}(r, t, b ; M) F\left(\frac{1}{2}-M, \frac{1}{2}-M ; 1 ; \gamma(r, t, b)\right) \\
& +C(r, t, b ; M) F\left(\frac{3}{2}-M, \frac{3}{2}-M ; 2 ; \gamma(r, t, b)\right) \\
& +D(r, t, b ; M) F\left(\frac{5}{2}-M, \frac{5}{2}-M ; 3 ; \gamma(r, t, b)\right),
\end{aligned}
$$

where

$$
\begin{aligned}
C(r, t, b ; M) & =A(r, t, b ; M) \gamma_{t}(r, t, b)\left(\frac{1}{2}-M\right)^{2}+B_{t}(r, t, b ; M) \\
D(r, t, b ; M) & =\frac{1}{2}\left(\frac{3}{2}-M\right)^{2} B(r, t, b ; M) \gamma_{t}(r, t, b) .
\end{aligned}
$$

Proof. It can be verified by simple calculations. Proposition is proven.

Corollary 1.11 The functions $A_{t}(r, t, b ; M), C(r, t, b ; M)$, and $D(r, t, b ; M)$, explicitly written, are as follows

$$
\begin{aligned}
& A_{t}(r, t, b ; M) \\
= & 4^{-M} M^{2} e^{M(b+t)}\left(\left(e^{-b}+e^{-t}\right)^{2}-r^{2}\right)^{M-\frac{1}{2}} \\
& -2^{-2 M}(2 M-1) e^{M(b+t)-b-2 t}\left(\left(e^{-b}+e^{-t}\right)^{2}-r^{2}\right)^{M-\frac{3}{2}}\left[2 e^{b} M-2 e^{b}+2 M e^{t}-e^{t}\right] \\
& +2^{2-2 M}\left(M-\frac{1}{2}\right)\left(M-\frac{3}{2}\right)\left(e^{-b}+e^{-t}\right)^{2} e^{M(b+t)-2 t}\left(\left(e^{-b}+e^{-t}\right)^{2}-r^{2}\right)^{M-\frac{5}{2}} \\
& C(r, t, b ; M)
\end{aligned}
$$




$$
\begin{aligned}
= & -2^{3-2 M}\left(\frac{1}{2}-M\right)^{2} M e^{M(b+t)-3 b-t}\left(\left(e^{-b}+e^{-t}\right)^{2}-r^{2}\right)^{M-\frac{5}{2}}\left[-e^{2 b} r^{2}-e^{2 b-2 t}+1\right] \\
& -2^{2-2 M}\left(\frac{1}{2}-M\right)^{2} e^{(M-5)(b+t)}\left(\left(e^{-b}+e^{-t}\right)^{2}-r^{2}\right)^{M-\frac{7}{2}} \\
& \times\left[-4 M r^{2} e^{3 b+3 t}-4 M r^{2} e^{4 b+2 t}+4 M e^{2(b+t)}-4 M e^{3 b+t}+4 M e^{b+3 t}-4 e^{4 b} M\right. \\
& \left.+r^{4} e^{4 b+4 t}+4 r^{2} e^{3 b+3 t}-2 r^{2} e^{2 b+4 t}+8 r^{2} e^{4 b+2 t}-8 e^{2(b+t)}-4 e^{b+3 t}+3 e^{4 b}+e^{4 t}\right], \\
& D(r, t, b ; M) \\
= & 2^{3-2 M}\left(\frac{1}{2}-M\right)^{2}\left(\frac{3}{2}-M\right)^{2} e^{M(b+t)-2 t}\left(\left(e^{-b}+e^{-t}\right)^{2}-r^{2}\right)^{M-\frac{9}{2}} \\
& \times\left[e^{-2 b} r^{4}+2 r^{2} e^{-2 b-2 t}-2 e^{-4 b} r^{2}-2 e^{-4 b-2 t}+e^{-2 b-4 t}+e^{-6 b}\right] .
\end{aligned}
$$

Theorem 1.12 The function $E(r, t ; 0, b ; M)$ solves the following equation

$$
E_{t t}(r, t ; 0, b ; M)-e^{-2 t} E_{r r}(r, t ; 0, b ; M)-M^{2} E(r, t ; 0, b ; M)=0 .
$$

Proof. We have

$$
\begin{aligned}
& E_{t t}(r, t ; 0, b ; M)-e^{-2 t} E_{r r}(r, t ; 0, b ; M)-M^{2} E(r, t ; 0, b ; M) \\
= & I(r, t, b ; M) F\left(\frac{1}{2}-M, \frac{1}{2}-M ; 1 ; \gamma(r, t, b)\right)+J(r, t, b ; M) F\left(\frac{3}{2}-M, \frac{3}{2}-M ; 2 ; \gamma(r, t, b)\right) \\
& +Y(r, t, b ; M) F\left(\frac{5}{2}-M, \frac{5}{2}-M ; 3 ; \gamma(r, t, b)\right),
\end{aligned}
$$

where

$$
\begin{aligned}
I(r, t, b ; M) & =A_{t}(r, t, b ; M)-e^{-2 t} \widetilde{A}(r, t, b ; M)-M^{2} \alpha(r, t, b ; M) \beta(r, t, b) \\
J(r, t, b ; M) & =C(r, t, b ; M)-e^{-2 t} \widetilde{B}(r, t, b ; M) \\
Y(r, t, b ; M) & =D(r, t, b ; M)-e^{-2 t} \widetilde{C}(r, t, b ; M) .
\end{aligned}
$$

That is,

$$
\begin{aligned}
I(r, t, b ; M)= & 4^{-M} M^{2} e^{M(b+t)}\left(\left(e^{-b}+e^{-t}\right)^{2}-r^{2}\right)^{M-\frac{1}{2}} \\
& -2^{-2 M}(2 M-1) e^{M(b+t)-b-2 t}\left(\left(e^{-b}+e^{-t}\right)^{2}-r^{2}\right)^{M-\frac{3}{2}}\left[2 e^{b} M-2 e^{b}+2 M e^{t}-e^{t}\right] \\
& +2^{2-2 M}\left(M-\frac{1}{2}\right)\left(M-\frac{3}{2}\right)\left(e^{-b}+e^{-t}\right)^{2} e^{M(b+t)-2 t}\left(\left(e^{-b}+e^{-t}\right)^{2}-r^{2}\right)^{M-\frac{5}{2}} \\
& -e^{-2 t}\left(2^{2-2 M}\left(M-\frac{3}{2}\right)\left(M-\frac{1}{2}\right) r^{2} e^{M(b+t)}\left(\left(e^{-b}+e^{-t}\right)^{2}-r^{2}\right)^{M-\frac{5}{2}}\right. \\
& \left.-2^{1-2 M}\left(M-\frac{1}{2}\right) e^{M(b+t)}\left(\left(e^{-b}+e^{-t}\right)^{2}-r^{2}\right)^{M-\frac{3}{2}}\right) .
\end{aligned}
$$

After simplification we obtain

$$
I(r, t, b ; M)=-4^{-M}(1-2 M)^{2} e^{(M-1)(b+t)}\left(\left(e^{-b}+e^{-t}\right)^{2}-r^{2}\right)^{M-\frac{3}{2}} .
$$

Then

$$
J(r, t, b ; M)=4^{-M}\left(\frac{1}{2}-M\right)^{2} e^{M(b+t)}\left(\left(e^{-b}+e^{-t}\right)^{2}-r^{2}\right)^{M-\frac{1}{2}}
$$




$$
\begin{aligned}
& \times\left(\frac{8 e^{-2 t}\left(e^{-b}+e^{-t}\right)^{2}\left(\left(e^{-b}-e^{-t}\right)^{2}-r^{2}\right)}{\left(\left(e^{-b}+e^{-t}\right)^{2}-r^{2}\right)^{3}}\right. \\
& +\frac{8 e^{-2 t}\left(e^{-b}-e^{-t}\right)\left(e^{-b}+e^{-t}\right)}{\left(\left(e^{-b}+e^{-t}\right)^{2}-r^{2}\right)^{2}}-\frac{2 e^{-2 t}\left(\left(e^{-b}-e^{-t}\right)^{2}-r^{2}\right)}{\left(\left(e^{-b}+e^{-t}\right)^{2}-r^{2}\right)^{2}} \\
& -\frac{2 e^{-t}\left(e^{-b}+e^{-t}\right)\left(\left(e^{-b}-e^{-t}\right)^{2}-r^{2}\right)}{\left(\left(e^{-b}+e^{-t}\right)^{2}-r^{2}\right)^{2}}+\frac{2 e^{-2 t}}{\left(e^{-b}+e^{-t}\right)^{2}-r^{2}} \\
& \left.-\frac{2 e^{-t}\left(e^{-b}-e^{-t}\right)}{\left(e^{-b}+e^{-t}\right)^{2}-r^{2}}\right) \\
& -2^{2-2 M}\left(\frac{1}{2}-M\right)^{2}\left(M-\frac{1}{2}\right)\left(e^{-b}+e^{-t}\right) e^{M(b+t)-t} \\
& \times\left(\left(e^{-b}+e^{-t}\right)^{2}-r^{2}\right)^{M-\frac{3}{2}} \\
& \times\left(\frac{2 e^{-t}\left(e^{-b}-e^{-t}\right)}{\left(e^{-b}+e^{-t}\right)^{2}-r^{2}}+\frac{2 e^{-t}\left(e^{-b}+e^{-t}\right)\left(\left(e^{-b}-e^{-t}\right)^{2}-r^{2}\right)}{\left(\left(e^{-b}+e^{-t}\right)^{2}-r^{2}\right)^{2}}\right) \\
& +2^{1-2 M}\left(\frac{1}{2}-M\right)^{2} M e^{M(b+t)}\left(\left(e^{-b}+e^{-t}\right)^{2}-r^{2}\right)^{M-\frac{1}{2}} \\
& \times\left(\frac{2 e^{-t}\left(e^{-b}-e^{-t}\right)}{\left(e^{-b}+e^{-t}\right)^{2}-r^{2}}+\frac{2 e^{-t}\left(e^{-b}+e^{-t}\right)\left(\left(e^{-b}-e^{-t}\right)^{2}-r^{2}\right)}{\left(\left(e^{-b}+e^{-t}\right)^{2}-r^{2}\right)^{2}}\right) \\
& -e^{-2 t}\left(4^{-M}\left(\frac{1}{2}-M\right)^{2} e^{M(b+t)}\left(\left(e^{-b}+e^{-t}\right)^{2}-r^{2}\right)^{M-\frac{1}{2}}\right. \\
& \times\left(\frac{8 r^{2}\left(\left(e^{-b}-e^{-t}\right)^{2}-r^{2}\right)}{\left(\left(e^{-b}+e^{-t}\right)^{2}-r^{2}\right)^{3}}-\frac{8 r^{2}}{\left(\left(e^{-b}+e^{-t}\right)^{2}-r^{2}\right)^{2}}+\right. \\
& \left.+\frac{2\left(\left(e^{-b}-e^{-t}\right)^{2}-r^{2}\right)}{\left(\left(e^{-b}+e^{-t}\right)^{2}-r^{2}\right)^{2}}-\frac{2}{\left(e^{-b}+e^{-t}\right)^{2}-r^{2}}\right) \\
& -2^{2-2 M}\left(\frac{1}{2}-M\right)^{2}\left(M-\frac{1}{2}\right) r e^{M(b+t)}\left(\left(e^{-b}+e^{-t}\right)^{2}-r^{2}\right)^{M-\frac{3}{2}} \\
& \left.\times\left(\frac{2 r\left(\left(e^{-b}-e^{-t}\right)^{2}-r^{2}\right)}{\left(\left(e^{-b}+e^{-t}\right)^{2}-r^{2}\right)^{2}}-\frac{2 r}{\left(e^{-b}+e^{-t}\right)^{2}-r^{2}}\right)\right) \text {. }
\end{aligned}
$$

After simplification we obtain

$$
\begin{aligned}
J(r, t, b ; M)= & -4^{-M}(1-2 M)^{2} e^{(M-3)(b+t)}\left(\left(e^{-b}+e^{-t}\right)^{2}-r^{2}\right)^{M-\frac{5}{2}} \\
& \times\left[2 M r^{2} e^{2(b+t)}+4 M e^{b+t}-2 e^{2 b} M-2 M e^{2 t}-r^{2} e^{2(b+t)}-6 e^{b+t}+e^{2 b}+e^{2 t}\right] .
\end{aligned}
$$


Then

$$
\begin{aligned}
Y(r, t, b ; M)= & 2^{-2 M-1}\left(\frac{1}{2}-M\right)^{2}\left(\frac{3}{2}-M\right)^{2} e^{M(b+t)}\left(\left(e^{-b}+e^{-t}\right)^{2}-r^{2}\right)^{M-\frac{1}{2}} \\
& \times\left(\frac{2 e^{-t}\left(e^{-b}-e^{-t}\right)}{\left(e^{-b}+e^{-t}\right)^{2}-r^{2}}+\frac{2 e^{-t}\left(e^{-b}+e^{-t}\right)\left(\left(e^{-b}-e^{-t}\right)^{2}-r^{2}\right)}{\left(\left(e^{-b}+e^{-t}\right)^{2}-r^{2}\right)^{2}}\right)^{2}- \\
& -2^{-2 M-1}\left(\frac{1}{2}-M\right)^{2}\left(\frac{3}{2}-M\right)^{2} e^{M(b+t)-2 t}\left(\left(e^{-b}+e^{-t}\right)^{2}-r^{2}\right)^{M-\frac{1}{2}} \\
& \times\left(\frac{2 r\left(\left(e^{-b}-e^{-t}\right)^{2}-r^{2}\right)}{\left(\left(e^{-b}+e^{-t}\right)^{2}-r^{2}\right)^{2}}-\frac{2 r}{\left(e^{-b}+e^{-t}\right)^{2}-r^{2}}\right)^{2}
\end{aligned}
$$

In fact, we obtain

$$
\begin{aligned}
Y(r, t, b ; M)= & 2^{3-2 M}\left(\frac{1}{2}-M\right)^{2}\left(\frac{3}{2}-M\right)^{2} e^{-2 t} e^{(M-2)(b+t)}\left(\left(e^{-b}+e^{-t}\right)^{2}-r^{2}\right)^{M-\frac{9}{2}} \\
& \times\left[-2 r^{2} e^{2 t-2 b}+e^{2 t-4 b}-2 e^{-2 b}+r^{4} e^{2 t}-2 r^{2}+e^{-2 t}\right] .
\end{aligned}
$$

Furthermore,

$$
\begin{aligned}
J(r, t, b ; M) F\left(\frac{3}{2}-M, \frac{3}{2}-M ; 2 ; \gamma(r, t, b)\right) & =\widetilde{J}(r, t, b ; M) F_{z}\left(\frac{1}{2}-M, \frac{1}{2}-M ; 1 ; \gamma(r, t, b)\right) \\
Y(r, t, b ; M) F\left(\frac{5}{2}-M, \frac{5}{2}-M ; 3 ; \gamma(r, t, b)\right) & =\widetilde{Y}(r, t, b ; M) F_{z z}\left(\frac{1}{2}-M, \frac{1}{2}-M ; 1 ; \gamma(r, t, b)\right)
\end{aligned}
$$

where $F_{z}(a, b ; 1 ; z):=\frac{d}{d z} F(a, b ; 1 ; z)$ and the following notations have been used

$$
\begin{aligned}
\widetilde{J}(r, t, b ; M) & =J(r, t, b ; M)\left(\frac{1}{2}-M\right)^{-2} \\
\widetilde{Y}(r, t, b ; M) & =Y(r, t, b ; M) 2\left(\frac{1}{2}-M\right)^{-2}\left(\frac{3}{2}-M\right)^{-2} .
\end{aligned}
$$

Hence

$$
\begin{aligned}
& E_{t t}(r, t ; 0, b ; M)-e^{-2 t} E_{r r}(r, t ; 0, b ; M)-M^{2} E(r, t ; 0, b ; M) \\
= & I(r, t, b ; M) F\left(\frac{1}{2}-M, \frac{1}{2}-M ; 1 ; \gamma(r, t, b)\right)+\widetilde{J}(r, t, b ; M) F_{z}\left(\frac{1}{2}-M, \frac{1}{2}-M ; 1 ; \gamma(r, t, b)\right) \\
& +\widetilde{Y}(r, t, b ; M) 2 F_{z z}\left(\frac{1}{2}-M, \frac{1}{2}-M ; 1 ; \gamma(r, t, b)\right) .
\end{aligned}
$$

Next, if we denote

$$
G(r, t, b ; M):=4^{1-M} e^{(M-1)(b+t)}\left(\left(e^{-b}+e^{-t}\right)^{2}-r^{2}\right)^{M-\frac{3}{2}},
$$

then

$$
\begin{aligned}
& I(r, t, b ; M) F\left(\frac{1}{2}-M, \frac{1}{2}-M ; 1 ; \gamma(r, t, b)\right)+\widetilde{J}(r, t, b ; M) F_{z}\left(\frac{1}{2}-M, \frac{1}{2}-M ; 1 ; \gamma(r, t, b)\right) \\
& +\widetilde{Y}(r, t, b ; M) F_{z z}\left(\frac{1}{2}-M, \frac{1}{2}-M ; 1 ; \gamma(r, t, b)\right)
\end{aligned}
$$




$$
\begin{aligned}
= & G(r, t, b ; M)\left\{\gamma(1-\gamma) F_{z z}\left(\frac{1}{2}-M, \frac{1}{2}-M ; 1 ; \gamma(r, t, b)\right)\right. \\
& +\left(1-\left(1+2\left(\frac{1}{2}-M\right)\right) \gamma\right) F_{z}\left(\frac{1}{2}-M, \frac{1}{2}-M ; 1 ; \gamma(r, t, b)\right) \\
& \left.-\left(\frac{1}{2}-M\right)^{2} F\left(\frac{1}{2}-M, \frac{1}{2}-M ; 1 ; \gamma(r, t, b)\right)\right\},
\end{aligned}
$$

since

$$
G(r, t, b ; M)=\frac{I(r, t, b ; M)}{-\left(\frac{1}{2}-M\right)^{2}}=\frac{\widetilde{J}(r, t, b ; M)}{\left(1-\left(2\left(\frac{1}{2}-M\right)+1\right) \gamma\right)}=\frac{\widetilde{Y}(r, t, b ; M)}{\gamma(1-\gamma)}
$$

The hypergeometric function $F\left(\frac{1}{2}-M, \frac{1}{2}-M ; 1 ; z\right)$ solves the following equation

$$
z(1-z) F_{z z}+\left(1-\left(2\left(\frac{1}{2}-M\right)+1\right) z\right) F_{z}-\left(\frac{1}{2}-M\right)^{2} F=0 .
$$

Theorem is proven.

\subsection{The kernel function $K_{1}(r, t ; M)$}

By definition

$$
K_{1}(r, t ; M):=4^{-M} e^{M t}\left(\left(1+e^{-t}\right)^{2}-r^{2}\right)^{-\frac{1}{2}+M} F\left(\frac{1}{2}-M, \frac{1}{2}-M ; 1 ; \frac{\left(1-e^{-t}\right)^{2}-r^{2}}{\left(1+e^{-t}\right)^{2}-r^{2}}\right),
$$

where $t \geq 0, r \in\left[0,1-e^{-t}\right]$, and $M \in \mathbb{C}$. In fact,

$$
K_{1}(r, t ; M)=E(r, t ; 0,0, M),
$$

which simplifies the proof of many properties of this function since they are inherited from the kernel $E(z, t ; 0, b, M)$.

Proposition 1.13 We have

$$
\begin{aligned}
& 2 e^{-t} K_{1 r}(\phi(t), t ; M)+2 K_{1 t}(\phi(t), t ; M)-K_{1}(\phi(t), t ; M)=0 \text { for all } t>0, \\
& K_{1 r}(0, t ; M)=0 \text { for all } t>0 .
\end{aligned}
$$

Proof. Due to Corollary 1.5, the derivative $\partial_{z} K_{1}(z, t ; M)$, explicitly written, is as follows

$$
\begin{aligned}
\partial_{r} K_{1}(r, t ; M)= & 2\left(\frac{1}{2}-M\right) r 4^{-M} e^{M t}\left(\left(e^{-t}+1\right)^{2}-r^{2}\right)^{M-\frac{3}{2}} \\
& {\left[F\left(\frac{1}{2}-M, \frac{1}{2}-M ; 1 ; \gamma(r, t, 0)\right)\right.} \\
& \left.\quad-\frac{4 e^{-t}}{\left(1+e^{-t}\right)^{2}-r^{2}}\left(\frac{1}{2}-M\right) F\left(\frac{3}{2}-M, \frac{3}{2}-M ; 2 ; \gamma(r, t, 0)\right)\right] .
\end{aligned}
$$

In particular, this proves the second equation of the proposition. Moreover, if $r=\phi(t)=1-e^{-t}$, then

$$
K_{1 r}\left(1-e^{-t}, t ; M\right)=\frac{1}{4}\left(\frac{1}{4}-M^{2}\right)\left(1-e^{-t}\right) e^{\frac{3}{2} t} .
$$


Then, due to Proposition 1.7 and Corollary 1.8, the derivative $\partial_{t} K_{1}(z, t ; M)$ is

$$
\begin{aligned}
\partial_{t} K_{1}(r, t ; M)= & A(r, t, 0 ; M) F\left(\frac{1}{2}-M, \frac{1}{2}-M ; 1 ; \gamma(r, t, 0)\right) \\
& +B(r, t, 0 ; M) F\left(\frac{3}{2}-M, \frac{3}{2}-M ; 2 ; \gamma(r, t, 0)\right)
\end{aligned}
$$

where

$$
A(r, t, 0 ; M)=4^{-M} e^{(M-2) t}\left(\left(e^{-t}+1\right)^{2}-z^{2}\right)^{M-\frac{3}{2}}\left(-M e^{2 t} r^{2}+M e^{2 t}-M+e^{t}+1\right)
$$

and

$$
B(r, t, 0 ; M)=-4^{1-M}\left(\frac{1}{2}-M\right)^{2} e^{(M-3) t}\left(e^{2 t}\left(r^{2}-1\right)+1\right)\left(\left(e^{-t}+1\right)^{2}-r^{2}\right)^{M-\frac{5}{2}}
$$

Hence,

$$
\begin{aligned}
K_{1 t}(r, t ; M)= & 4^{-M} e^{(M-2) t}\left(\left(e^{-t}+1\right)^{2}-r^{2}\right)^{M-\frac{3}{2}}\left(-M e^{2 t} r^{2}+M e^{2 t}-M+e^{t}+1\right) \\
& \times F\left(\frac{1}{2}-M, \frac{1}{2}-M ; 1 ; \gamma(r, t, 0)\right) \\
& -4^{1-M}\left(\frac{1}{2}-M\right)^{2} e^{(M-3) t}\left(e^{2 t}\left(r^{2}-1\right)+1\right)\left(\left(e^{-t}+1\right)^{2}-r^{2}\right)^{M-\frac{5}{2}} \\
& \times F\left(\frac{3}{2}-M, \frac{3}{2}-M ; 2 ; \gamma(r, t, 0)\right) .
\end{aligned}
$$

In particular,

$$
K_{1 t}\left(1-e^{-t}, t ; M\right)=\frac{1}{16} e^{-\frac{1}{2} t}\left(\left(4 M^{2}+3\right) e^{t}-4 M^{2}+1\right)
$$

while

$$
K_{1}\left(1-e^{-t}, t ; M\right)=\frac{1}{2} e^{\frac{1}{2} t}
$$

This completes the proof of the proposition.

Furthermore, the following proposition easily follows from Theorem 1.12.

Proposition 1.14 The function solves the following equation

$$
K_{1 t t}(r, t ; M)-e^{-2 t} K_{1 r r}(r, t ; M)-M^{2} K_{1}(r, t ; M)=0 \quad \text { for all } \quad t>0, \quad 0<r<t .
$$

\subsection{The kernel function $K_{0}(r, t ; M)$}

The function $K_{0}(r, t ; M)$ can be written as follows

$$
K_{0}(r, t ; M)=-\left[\frac{\partial}{\partial b} E(r, t ; 0, b ; M)\right]_{b=0} .
$$

We consider $K_{0}(r, t ; M)$ for $r \in\left[0,1-e^{-t}\right)$, and $M \in \mathbb{C}$. We have

$$
K_{0}(\phi(t), t ; M):=\lim _{r \rightarrow \phi(t)} K_{0}(r, t ; M)=-\frac{1}{4} M^{2} e^{t \frac{1}{2}}+\frac{1}{4} M^{2} e^{\frac{3}{2} t}-\frac{3}{16} e^{\frac{1}{2} t}-\frac{1}{16} e^{\frac{3}{2} t} .
$$


Then

$$
\begin{aligned}
& \partial_{r} K_{0}(r, t ; M)=K_{0 r}(r, t ; M) \\
= & \frac{1}{\left(e^{t}\left(\left(r^{2}-1\right) e^{t}+2\right)-1\right)^{2}} 4^{-M}(2 M-1) r e^{M t}\left(\left(1+e^{-t}\right)^{2}-r^{2}\right)^{M-\frac{3}{2}} \\
& \times\left\{\left[M r^{4} e^{4 t}+2 M r^{2} e^{3 t}-2 M r^{2} e^{4 t}+2 M e^{t}-2 M e^{3 t}+M e^{4 t}-M-3 r^{2} e^{2 t}\right.\right. \\
& \left.+r^{2} e^{3 t}+e^{t}-3 e^{2 t}-e^{3 t}+3\right] F\left(\frac{1}{2}-M, \frac{1}{2}-M ; 1 ; \gamma(r, t, 0)\right) \\
& \left.-\frac{2 r^{4} e^{5 t}+12 r^{2} e^{3 t}-4 r^{2} e^{5 t}-14 e^{t}+12 e^{3 t}+2 e^{5 t}}{e^{t}\left(\left(r^{2}-1\right) e^{t}-2\right)-1} F\left(\frac{1}{2}-M, \frac{3}{2}-M ; 1 ; \gamma(r, t, 0)\right)\right\} .
\end{aligned}
$$

Thus,

Similarly,

$$
\begin{aligned}
K_{0 r}(\phi(t), t ; M):=\lim _{r \rightarrow \phi(t)} K_{0 r}(r, t ; M)= & -\frac{1}{16} M^{4} e^{\frac{1}{2} t}+\frac{1}{8} M^{4} e^{\frac{3}{2} t}-\frac{1}{16} M^{4} e^{\frac{5}{2} t}-\frac{7}{32} M^{2} e^{\frac{1}{2} t} \\
& +\frac{1}{16} M^{2} e^{\frac{3}{2} t}+\frac{5}{32} M^{2} e^{\frac{5}{2} t}+\frac{15}{256} e^{\frac{1}{2} t}-\frac{3}{128} e^{\frac{3}{2} t}-\frac{9}{256} e^{\frac{5}{2} t} .
\end{aligned}
$$

$$
\begin{aligned}
& K_{0 t}(r, t ; M) \\
= & \frac{4^{-M} e^{(M-2) t}\left(-r^{2}+e^{-2 t}+2 e^{-t}+1\right)^{M-\frac{3}{2}}}{\left(e^{t}\left(\left(r^{2}-1\right) e^{t}+2\right)-1\right)^{2}} \\
& \times\left\{\left[M^{2} r^{6} e^{6 t}-M^{2} r^{4} e^{4 t}+2 M^{2} r^{4} e^{5 t}-M^{2} r^{4} e^{6 t}-M^{2} r^{2} e^{2 t}+2 M^{2} r^{2} e^{4 t}\right.\right. \\
& -M^{2} r^{2} e^{6 t}-2 M^{2} e^{t}-M^{2} e^{2 t}+4 M^{2} e^{3 t}-M^{2} e^{4 t}-2 M^{2} e^{5 t}+M^{2} e^{6 t}+M^{2}-M r^{4} e^{4 t} \\
& -M r^{4} e^{6 t}+2 M r^{2} e^{2 t}-12 M r^{2} e^{4 t}+2 M r^{2} e^{6 t}+M e^{2 t}+M e^{4 t}-M e^{6 t}-M-r^{2} e^{3 t} \\
& \left.+6 r^{2} e^{4 t}-r^{2} e^{5 t}+e^{t}-2 e^{3 t}+e^{5 t}\right] F\left(\frac{1}{2}-M, \frac{1}{2}-M ; 1 ; \frac{\left(1-e^{-t}\right)^{2}-r^{2}}{\left(e^{-t}+1\right)^{2}-r^{2}}\right) \\
& -\frac{1}{e^{t}\left(\left(r^{2}-1\right) e^{t}-2\right)-1}\left[4 M r^{4} e^{5 t}+4 M r^{4} e^{7 t}-8 M r^{2} e^{3 t}+48 M r^{2} e^{5 t}\right. \\
& -8 M r^{2} e^{7 t}+4 M e^{t}-4 M e^{3 t}-4 M e^{5 t}+4 M e^{7 t}-2 r^{4} e^{5 t}-2 r^{4} e^{7 t}+4 r^{2} e^{3 t} \\
& \left.\left.-24 r^{2} e^{5 t}+4 r^{2} e^{7 t}-2 e^{t}+2 e^{3 t}+2 e^{5 t}-2 e^{7 t}\right] F\left(\frac{1}{2}-M, \frac{3}{2}-M ; 1 ; \frac{\left(1-e^{-t}\right)^{2}-r^{2}}{\left(e^{-t}+1\right)^{2}-r^{2}}\right)\right\}
\end{aligned}
$$

and

$$
\begin{aligned}
K_{0 t}(\phi(t), t ; M):= & \lim _{r \rightarrow \phi(t)} K_{0 t}(r, t ; M) \\
= & \frac{1}{16} M^{4} e^{-\frac{1}{2} t}-\frac{1}{8} M^{4} e^{\frac{1}{2} t}+\frac{1}{16} M^{4} e^{\frac{3}{2} t} \\
& +\frac{7}{32} M^{2} e^{-\frac{1}{2} t}-\frac{3}{16} M^{2} e^{\frac{1}{2} t}+\frac{7}{32} M^{2} e^{\frac{3}{2} t}+\frac{15}{256} e^{-\frac{1}{2} t}-\frac{9}{128} e^{\frac{1}{2} t}-\frac{15}{256} e^{\frac{3}{2} t}
\end{aligned}
$$

Proposition 1.15 For all $M$ we have

$$
\begin{aligned}
& K_{0 r}(0, t ; M)=0, \quad \text { for all } t>0, \\
& K_{0 t t}(r, t ; M)-e^{-2 t} K_{0 r r}(r, t ; M)-M^{2} K_{0}(r, t ; M)=0 \quad \text { for all } t>0, \quad r \in\left[0,1-e^{-t}\right], \\
& \left(\frac{1}{4}-M^{2}\right) e^{\frac{t}{2}}-2 e^{-t} K_{0}(\phi(t), t ; M)+4 e^{-2 t} K_{0 r}(\phi(t), t ; M)+4 e^{-t} K_{0 t}(\phi(t), t ; M)=0 .
\end{aligned}
$$


Proof. The first and second statements follow from the corresponding ones for the function $E$. Then, according to (1.3), (1.4), and (1.5), we have

$$
\begin{aligned}
& -2 e^{-t} K_{0}(\phi(t), t ; M)+4 e^{-2 t} K_{0 s}(\phi(t), t ; M)+4 e^{-t} K_{0 t}(\phi(t), t ; M) \\
= & -2 e^{-t}\left[-\frac{1}{4} M^{2} e^{t \frac{1}{2}}+\frac{1}{4} M^{2} e^{\frac{3}{2} t}-\frac{3}{16} e^{\frac{1}{2} t}-\frac{1}{16} e^{\frac{3}{2} t}\right] \\
& +4 e^{-2 t}\left[-\frac{1}{16} M^{4} e^{\frac{1}{2} t}+\frac{1}{8} M^{4} e^{\frac{3}{2} t}-\frac{1}{16} M^{4} e^{\frac{5}{2} t}-\frac{7}{32} M^{2} e^{\frac{1}{2} t}\right. \\
& \left.+\frac{1}{16} M^{2} e^{\frac{3}{2} t}+\frac{5}{32} M^{2} e^{\frac{5}{2} t}+\frac{15}{256} e^{\frac{1}{2} t}-\frac{3}{128} e^{\frac{3}{2} t}-\frac{9}{256} e^{\frac{5}{2} t}\right] \\
& +4 e^{-t}\left[\frac{1}{16} M^{4} e^{-\frac{1}{2} t}-\frac{1}{8} M^{4} e^{\frac{1}{2} t}+\frac{1}{16} M^{4} e^{\frac{3}{2} t}\right. \\
& \left.+\frac{7}{32} M^{2} e^{-\frac{1}{2} t}-\frac{3}{16} M^{2} e^{\frac{1}{2} t}+\frac{7}{32} M^{2} e^{\frac{3}{2} t}-\frac{15}{256} e^{-\frac{1}{2} t}-\frac{9}{128} e^{\frac{1}{2} t}-\frac{15}{256} e^{\frac{3}{2} t}\right] \\
= & -\left(\frac{1}{4}-M^{2}\right) e^{\frac{t}{2}} .
\end{aligned}
$$

This proves the last statement. Proposition is proven.

\section{Proof of Theorem 0.1}

The proof is straightforward; we just substitute the function $u=u(t, x)(0.11)$ into equation (0.12) and then check the initial conditions. In order to make proof more transparent we split it into three independent cases.

Case of $\left(\varphi_{1}\right)$ In this case set $f(t, x)=0, \varphi_{0}(x)=0$. Henceforth we suppress the subindex $\varphi_{1}$ of $v_{\varphi_{1}}$. We have

$$
\frac{1}{2} u(x, t)=\int_{0}^{\phi(t)} v(x, s) K_{1}(s, t ; M) d s, \quad x \in \mathbb{R}^{n}, t>0,
$$

and consider the derivative

$$
\begin{aligned}
& \frac{1}{2} \partial_{t}^{2} u(x, t) \\
= & -e^{-t} v(x, \phi(t)) K_{1}(\phi(t), t ; M)+e^{-2 t} v_{t}(x, \phi(t)) K_{1}(\phi(t), t ; M)+e^{-2 t} v(x, \phi(t)) K_{1 s}(\phi(t), t ; M) \\
& +2 e^{-t} v(x, \phi(t)) K_{1 t}(\phi(t), t ; M)+\int_{0}^{\phi(t)} v(x, s) K_{1 t t}(s, t ; M) d s .
\end{aligned}
$$

According to the choice (0.10) of the function $v$, we have

$$
\frac{1}{2} A\left(x, \partial_{x}\right) u(x, t)=\int_{0}^{\phi(t)} A\left(x, \partial_{x}\right) v(x, s) K_{1}(s, t ; M) d s=\int_{0}^{\phi(t)} v_{t t}(x, s) K_{1}(s, t ; M) d s .
$$

Now we take into account $(0.10)$, that is $v_{t}(x, 0)=0$, and the second statement $K_{1 s}(0, t ; M)=0$ of Proposition 1.13. Hence we have

$$
\frac{1}{2} A\left(x, \partial_{x}\right) u(x, t)=v_{t}(x, \phi(t)) K_{1}(\phi(t), t ; M)-v(x, \phi(t)) K_{1 s}(\phi(t), t ; M)+\int_{0}^{\phi(t)} v(x, s) K_{1 s s}(s, t ; M) d s .
$$


Due to Proposition 1.13 and Proposition 1.14 we can write

$$
\begin{aligned}
& \partial_{t}^{2} u(x, t)-e^{-2 t} A\left(x, \partial_{x}\right) u(x, t)-M^{2} u(x, t) \\
= & 2 e^{-t} v(x, \phi(t))\left(-K_{1}(\phi(t), t ; M)+2 e^{-t} K_{1 s}(\phi(t), t ; M)+2 K_{1 t}(\phi(t), t ; M)\right) \\
& +2 \int_{0}^{\phi(t)} v(x, s)\left(K_{1 t t}(s, t ; M) d s-e^{-2 t} K_{1 s s}(s, t ; M) d s-M^{2} K_{1}(s, t ; M)\right) d s .
\end{aligned}
$$

This competes the proof of the case $\left(\varphi_{1}\right)$. Similarly, we consider the next case.

Case of $\left(\varphi_{0}\right)$ In this case we set $f(t, x)=0, \varphi_{1}(x)=0$, then (0.11) reads

$$
u(x, t)=e^{\frac{t}{2}} v_{\varphi_{0}}(x, \phi(t))+2 \int_{0}^{\phi(t)} v_{\varphi_{0}}(x, s) K_{0}(s, t ; M) d s .
$$

From now on we skip subindex $\varphi_{0}$ of $v_{\varphi_{0}}$. For the first and second-oder derivatives we have

$$
\begin{aligned}
\partial_{t} u(x, t)= & \frac{1}{2} e^{\frac{t}{2}} v(x, \phi(t))+e^{-\frac{t}{2}} v_{t}(x, \phi(t)) \\
& +2 e^{-t} v(x, \phi(t)) K_{0}(\phi(t), t ; M)+2 \int_{0}^{\phi(t)} v(x, s) K_{0 t}(s, t ; M) d s
\end{aligned}
$$

and

$$
\begin{aligned}
\partial_{t}^{2} u(x, t)= & \frac{1}{4} e^{\frac{t}{2}} v(x, \phi(t))+e^{-\frac{3 t}{2}} v_{t t}(x, \phi(t)) \\
& -2 e^{-t} v(x, \phi(t)) K_{0}(\phi(t), t ; M)+2 e^{-\frac{3 t}{2}} v_{t}(x, \phi(t)) K_{0}(\phi(t), t ; M) \\
& +2 e^{-\frac{3 t}{2}} v(x, \phi(t)) K_{0 s}(\phi(t), t ; M)+2 e^{-t} v(x, \phi(t)) K_{0 t}(\phi(t), t ; M) \\
& +2 e^{-t} v(x, \phi(t)) K_{0 t}(\phi(t), t ; M)+2 \int_{0}^{\phi(t)} v(x, s) K_{0 t t}(s, t ; M) d s,
\end{aligned}
$$

respectively, while

$$
A\left(x, \partial_{x}\right) u(x, t)=e^{\frac{t}{2}} A\left(x, \partial_{x}\right) v(x, \phi(t))+2 \int_{0}^{\phi(t)} v_{t t}(x, s) K_{0}(s, t ; M) d s .
$$

Consider the last integral; using twice integration by parts and the choice of function $v$, we obtain

$$
\begin{aligned}
\int_{0}^{\phi(t)} v_{s s}(x, s) K_{0}(s, t ; M) d s= & v_{s}(x, \phi(t)) K_{0}(\phi(t), t ; M)-v(x, \phi(t)) K_{0 s}(\phi(t), t ; M) \\
& +v(x, 0) K_{0 s}(0, t ; M)+\int_{0}^{\phi(t)} v(x, s) K_{0 s s}(s, t ; M) d s .
\end{aligned}
$$

Since $K_{0 s}(0, t ; M)=0$, we obtain

$$
\begin{aligned}
A\left(x, \partial_{x}\right) u(x, t)= & e^{\frac{t}{2}} v_{t t}(x, \phi(t))+2 v_{t}(x, \phi(t)) K_{0}(\phi(t), t ; M)-2 v(x, \phi(t)) K_{0 s}(\phi(t), t ; M) \\
& +2 \int_{0}^{\phi(t)} v(x, s) K_{0 s s}(s, t ; M) d s .
\end{aligned}
$$

Hence

$$
\begin{aligned}
& \partial_{t}^{2} u(x, t)-e^{-2 t} A\left(x, \partial_{x}\right) u(x, t)-M^{2} u(x, t) \\
= & v(x, \phi(t))\left[\left(\frac{1}{4}-M^{2}\right) e^{\frac{t}{2}}-2 e^{-t} K_{0}(\phi(t), t ; M)+2 e^{-2 t} K_{0 s}(\phi(t), t ; M)\right. \\
& \left.+2 e^{-t} v(x, \phi(t)) K_{0 t}(\phi(t), t ; M)+2 e^{-t} K_{0 t}(\phi(t), t ; M)+2 e^{-2 t} K_{0 s}(\phi(t), t ; M)\right] \\
& +2 \int_{0}^{\phi(t)} v(x, s)\left[K_{0 t t}(s, t ; M) d s-e^{-2 t} K_{0 s s}(s, t ; M) d s-M^{2} K_{0}(s, t ; M)\right] d s .
\end{aligned}
$$


Then we use Proposition 1.15, which completes the proof of the case of $\left(\varphi_{0}\right)$.

Case of (f) Similarly, we set $\varphi_{0}(x)=0, \varphi_{1}(x)=0$, then (0.11) reads

$$
u(x, t)=2 \int_{0}^{t} d b \int_{0}^{e^{-b}-e^{-t}} v(x, r ; b) E(r, t ; 0, b ; M) d r .
$$

For the first and second-order derivatives we obtain

$$
\begin{aligned}
\frac{1}{2} \partial_{t} u(x, t)= & e^{-t} \int_{0}^{t} v\left(x, e^{-b}-e^{-t} ; b\right) E\left(e^{-b}-e^{-t}, t ; 0, b ; M\right) d b \\
& +\int_{0}^{t} d b \int_{0}^{e^{-b}-e^{-t}} v(x, r ; b) E_{t}(r, t ; 0, b ; M) d r
\end{aligned}
$$

and

$$
\begin{aligned}
\frac{1}{2} \partial_{t}^{2} u(x, t)= & -e^{-t} \int_{0}^{t} v\left(x, e^{-b}-e^{-t} ; b\right) E\left(e^{-b}-e^{-t}, t ; 0, b ; M\right) d b \\
& +e^{-t} v(x, 0 ; t) E(0, t ; 0, t ; M) \\
& +e^{-2 t} \int_{0}^{t} v_{t}\left(x, e^{-b}-e^{-t} ; b\right) E\left(e^{-b}-e^{-t}, t ; 0, b ; M\right) d b \\
& +e^{-2 t} \int_{0}^{t} v\left(x, e^{-b}-e^{-t} ; b\right) E_{r}\left(e^{-b}-e^{-t}, t ; 0, b ; M\right) d b \\
& +2 e^{-t} \int_{0}^{t} v\left(x, e^{-b}-e^{-t} ; b\right) E_{t}\left(e^{-b}-e^{-t}, t ; 0, b ; M\right) d b \\
& +\int_{0}^{t} d b \int_{0}^{e^{-b}-e^{-t}} v(x, r ; b) E_{t t}(r, t ; 0, b ; M) d r
\end{aligned}
$$

respectively. On the other hand, due to the definition of the kernel $E$, Lemma 1.3, and (1.1), we obtain

$$
E\left(e^{-b}-e^{-t}, t ; 0, b ; M\right)=\frac{1}{2} e^{\frac{1}{2}(b+t)}, \quad E(0, t ; 0, t ; M)=\frac{1}{2} e^{t} .
$$

Hence, since $v(x, 0 ; t)=f(x, t)$, we have

$$
\begin{aligned}
\frac{1}{2} \partial_{t}^{2} u(x, t)= & -e^{-t} \int_{0}^{t} v\left(x, e^{-b}-e^{-t} ; b\right)\left(4 e^{-b-t}\right)^{-\frac{1}{2}} d b+\frac{1}{2} f(x, t) \\
& +e^{-2 t} \int_{0}^{t} v_{t}\left(x, e^{-b}-e^{-t} ; b\right)\left(4 e^{-b-t}\right)^{-\frac{1}{2}} d b \\
& +e^{-2 t} \int_{0}^{t} v\left(x, e^{-b}-e^{-t} ; b\right) E_{r}\left(e^{-b}-e^{-t}, t ; 0, b ; M\right) d b \\
& +2 e^{-t} \int_{0}^{t} v\left(x, e^{-b}-e^{-t} ; b\right) E_{t}\left(e^{-b}-e^{-t}, t ; 0, b ; M\right) d b \\
& +\int_{0}^{t} d b \int_{0}^{e^{-b}-e^{-t}} v(x, r ; b) E_{t t}(r, t ; 0, b ; M) d r .
\end{aligned}
$$

Then, taking into account (0.9), we have

$$
\begin{aligned}
\frac{1}{2} A\left(x, \partial_{x}\right) u(x, t)= & \int_{0}^{t} d b\left\{v_{r}\left(x, e^{-b}-e^{-t} ; b\right) E\left(e^{-b}-e^{-t}, t ; 0, b ; M\right)\right. \\
& \left.-v_{r}(x, 0 ; b) E(0, t ; 0, b ; M)-\int_{0}^{e^{-b}-e^{-t}} v_{r}(x, r ; b) E_{r}(r, t ; 0, b ; M) d r\right\} .
\end{aligned}
$$


The definition of $v,(0.11)$, and (2.1) imply

$$
\frac{1}{2} A\left(x, \partial_{x}\right) u(x, t)=\int_{0}^{t} d b\left\{v_{t}\left(x, e^{-b}-e^{-t} ; b\right)\left(4 e^{-b-t}\right)^{-\frac{1}{2}}-\int_{0}^{e^{-b}-e^{-t}} v_{r}(x, r ; b) E_{r}(r, t ; 0, b ; M) d r\right\} .
$$

Hence

$$
\begin{aligned}
& \frac{1}{2}\left(\partial_{t}^{2} u(x, t)-e^{-2 t} A\left(x, \partial_{x}\right) u(x, t)-M^{2} u(x, t)\right) \\
= & -e^{-t} \int_{0}^{t} v\left(x, e^{-b}-e^{-t} ; b\right)\left(4 e^{-b-t}\right)^{-\frac{1}{2}} d b+\frac{1}{2} f(x, t) \\
& +e^{-2 t} \int_{0}^{t} v\left(x, e^{-b}-e^{-t} ; b\right) E_{r}\left(e^{-b}-e^{-t}, t ; 0, b ; M\right) d b \\
& +2 e^{-t} \int_{0}^{t} v\left(x, e^{-b}-e^{-t} ; b\right) E_{t}\left(e^{-b}-e^{-t}, t ; 0, b ; M\right) d b \\
& +e^{-2 t} \int_{0}^{t} d b \int_{0}^{e^{-b}-e^{-t}} v_{r}(x, r ; b) E_{r}(r, t ; 0, b ; M) d r \\
& +\int_{0}^{t} d b \int_{0}^{e^{-b}-e^{-t}} v(x, r ; b)\left\{E_{t t}(r, t ; 0, b ; M)-M^{2} E(r, t ; 0, b ; M)\right\} d r
\end{aligned}
$$

On the other hand, the integration by parts leads to

$$
\begin{aligned}
& \int_{0}^{t} d b \int_{0}^{e^{-b}-e^{-t}} v_{r}(x, r ; b) E_{r}(r, t ; 0, b ; M) d r \\
= & \int_{0}^{t} d b\left\{v\left(x, e^{-b}-e^{-t} ; b\right) E_{r}\left(e^{-b}-e^{-t}, t ; 0, b ; M\right)-v(x, 0 ; b) E_{r}(0, t ; 0, b ; M)\right. \\
& \left.-\int_{0}^{e^{-b}-e^{-t}} v(x, r ; b) E_{r r}(r, t ; 0, b ; M) d r\right\}
\end{aligned}
$$

Then we use Proposition 1.4. Consequently,

$$
\begin{aligned}
& \int_{0}^{t} d b \int_{0}^{e^{-b}-e^{-t}} v_{r}(x, r ; b) E_{r}(r, t ; 0, b ; M) d r \\
= & \int_{0}^{t} d b\left\{v\left(x, e^{-b}-e^{-t} ; b\right) E_{r}\left(e^{-b}-e^{-t}, t ; 0, b ; M\right)-\int_{0}^{e^{-b}-e^{-t}} v(x, r ; b) E_{r r}(r, t ; 0, b ; M) d r\right\} .
\end{aligned}
$$

Thus,

$$
\begin{aligned}
& \frac{1}{2}\left(\partial_{t}^{2} u(x, t)-e^{-2 t} A\left(x, \partial_{x}\right) u(x, t)-M^{2} u(x, t)\right) \\
= & f(x, t) \frac{1}{2}-e^{-t} \int_{0}^{t} v\left(x, e^{-b}-e^{-t} ; b\right)\left(4 e^{-b-t}\right)^{-\frac{1}{2}} d b \\
& +e^{-2 t} \int_{0}^{t} v\left(x, e^{-b}-e^{-t} ; b\right) E_{r}\left(e^{-b}-e^{-t}, t ; 0, b ; M\right) d b \\
& +2 e^{-t} \int_{0}^{t} v\left(x, e^{-b}-e^{-t} ; b\right) E_{t}\left(e^{-b}-e^{-t}, t ; 0, b ; M\right) d b \\
& +e^{-2 t} \int_{0}^{t} d b v\left(x, e^{-b}-e^{-t} ; b\right) E_{r}\left(e^{-b}-e^{-t}, t ; 0, b ; M\right) \\
& +\int_{0}^{t} d b \int_{0}^{e^{-b}-e^{-t}} v(x, r ; b)\left\{E_{t t}(r, t ; 0, b ; M)-e^{-2 t} E_{r r}(r, t ; 0, b ; M)-M^{2} E(r, t ; 0, b ; M)\right\} d r
\end{aligned}
$$


Now we apply Theorem 1.12 to the last integral and obtain

$$
\begin{aligned}
& \frac{1}{2}\left(\partial_{t}^{2} u(x, t)-e^{-2 t} A\left(x, \partial_{x}\right) u(x, t)-M^{2} u(x, t)\right) \\
= & \frac{1}{2} f(x, t)+e^{-2 t} \int_{0}^{t} v\left(x, e^{-b}-e^{-t} ; b\right) \\
& \times\left\{2 E_{r}\left(e^{-b}-e^{-t}, t ; 0, b ; M\right)+2 e^{t} E_{t}\left(e^{-b}-e^{-t}, t ; 0, b ; M\right)-e^{t}\left(4 e^{-b-t}\right)^{-\frac{1}{2}}\right\} d b .
\end{aligned}
$$

Proposition 1.9 completes the proof of this case. It is easy to check the initial conditions. Theorem 0.1 is proven.

\section{References}

[1] A. Bachelot, On the Klein-Gordon equation near a De Sitter brane in an Anti-de Sitter bulk, arXiv:1402.1071v1.

[2] D. Baskin, A parametrix for the fundamental solution of the Klein-Gordon equation on asymptotically de Sitter spaces, Journal of Functional Analysis 259 (2010), 1673-1719.

[3] D. Baskin, Strichartz Estimates on Asymptotically de Sitter Spaces, Annales Henri Poincaré, 14, Issue 2 (2013) 221-252.

[4] H. Bateman, A. Erdelyi, Higher Transcendental Functions, v.1,2, McGraw-Hill, New York, 1953.

[5] N. D. Birrell, P.C.W. Davies, Quantum fields in curved space, Cambridge ; New York : Cambridge University Press, 1984.

[6] J. Bros, H. Epstein, U. Moschella, Particle decays and stability on the de Sitter universe, Ann. Henri Poincaré 11 (2010) no. 4, 611-658.

[7] Y. Choquet-Bruhat, P.T. Chrusciel, J.M. Martín-García, The light-cone theorem, Classical Quantum Gravity 26 (2009), no. 13, 135011, 22 pp.

[8] J.L. Costa, A. Alho, J. Natário, Spherical linear waves in de Sitter spacetime, J. Math. Phys. 53 (2012) no. 5, 052501, 9 pp.

[9] M. Dohse, Classical Klein-Gordon solutions, symplectic structures, and isometry actions on AdS spacetimes, $J$. Geom. Phys. 70 (2013) 130-156.

[10] A. Galstian, T. Kinoshita, K. Yagdjian, A note on wave equation in Einstein and de Sitter space-time, J. Math. Phys. 51, no. 5 (2010), 052501.

[11] A. Galstian, T. Kinoshita, Representation of Solutions for 2nd Order One-dimensional Model Hyperbolic Equations, Journal d'Analyse Mathematique, accepted for publication.

[12] S. W. Hawking, G. F. R. Ellis, The large scale structure of space-time, Cambridge Monographs on Mathematical Physics, No. 1. London-New York: Cambridge University Press, 1973.

[13] S. Helgason, Wave equations on homogeneous spaces. Lie group representations, III (College Park, Md., 1982/1983), 254-287, Lecture Notes in Math., 1077, Springer, Berlin, 1984.

[14] P. Hintz, Global well-posedness of quasilinear wave equations on asymptotically de Sitter spaces, arXiv:1311.6859v2.

[15] P. Hintz, A. Vasy, Semilinear wave equations on asymptotically de Sitter, Kerr-de Sitter and Minkowski spacetimes, arXiv:1306.4705v2.

[16] S. Jamal, A.H. Kara, and A.H. Bokhari, Symmetries, conservation laws, reductions, and exact solutions for the Klein-Gordon equation in de Sitter space-times, Can. J. Phys. 90 (2012) 667-674.

[17] De-Xing Kong, Chang-Hua Wei, Lifespan of smooth solutions for timelike extremal surface equation in de Sitter spacetime, arXiv:1311.3459v1.

[18] P. Lax, R.S. Phillips, Translation representations for the solution of the non-Euclidean wave equation. Comm. Pure Appl. Math. 32 no. 5 (1979) 617-667

[19] U. Moschella, The de Sitter and anti-de Sitter sightseeing tour. Einstein, 1905-2005, 120-133, Prog. Math. Phys., 47, Birkhäuser, Basel, 2006. 
[20] J. Näf, P. Jetzer, and M. Sereno, On gravitational waves in spacetimes with a nonvanishing cosmological constant, Phys. Rev. D 79 (2009) 024014.

[21] M. Nakamura, The Cauchy problem for semi-linear Klein-Gordon equations in de Sitter spacetime, J. Math. Anal. Appl. 410 no. 1 (2014) 445-454.

[22] L.E. Parker, D. J. Toms, Quantum field theory in curved spacetime, Quantized fields and gravity, Cambridge Monographs on Mathematical Physics, Cambridge University Press, Cambridge, 2009.

[23] A. Rendall, Asymptotics of solutions of the Einstein equations with positive cosmological constant, Ann. Henri Poincaré 5, (6) (2004) 1041-1064.

[24] W.A. Strauss, Partial differential equations. An introduction, Second edition, John Wiley \& Sons, Ltd., Chichester, 2008.

[25] R.C. Tolman, Relativity, thermodynamics, and cosmology, Clarendon Press, Oxford, 1934.

[26] A. Vasy, The wave equation on asymptotically de Sitter-like spaces. Adv. Math. 223, no. 1 (2010) 49-97.

[27] A. Vasy, Microlocal analysis of asymptotically hyperbolic and Kerr-de Sitter spaces (with an appendix by Semyon Dyatlov), Invent. Math. 194 no. 2 (2013) 381-513.

[28] K. Yagdjian, A note on the fundamental solution for the Tricomi-type equation in the hyperbolic domain, $J$. Differential Equations 206 (2004) 227-252.

[29] K. Yagdjian, A. Galstian, Fundamental Solutions for the Klein-Gordon Equation in de Sitter Spacetime, Comm. Math. Phys. 285 (2009) 293-344.

[30] K. Yagdjian, The semilinear Klein-Gordon equation in de Sitter spacetime, Discrete Contin. Dyn. Syst. Ser. S 2, no. 3 (2009) 679-696.

[31] K. Yagdjian, Fundamental Solutions for Hyperbolic Operators with Variable Coeffcients, Rend. Istit. Mat. Univ. Trieste 42 Suppl. (2010) 221-243.

[32] K. Yagdjian, On the global solutions of the Higgs boson equation, Comm. Partial Differential Equations 37, no. 3 (2012) 447-478.

[33] K. Yagdjian, Huygens' Principle for the Klein-Gordon equation in the de Sitter spacetime, J. Math. Phys. 54, no. 9 (2013) 091503.

[34] K. Yagdjian, Semilinear Hyperbolic Equations in Curved Spacetime. Fourier Analysis, Pseudo-differential Operators, Time-Frequency Analysis and Partial Differential Equations. Series: Trends in Mathematics. Birkhäuser Mathematics, 391-415, 2014. 